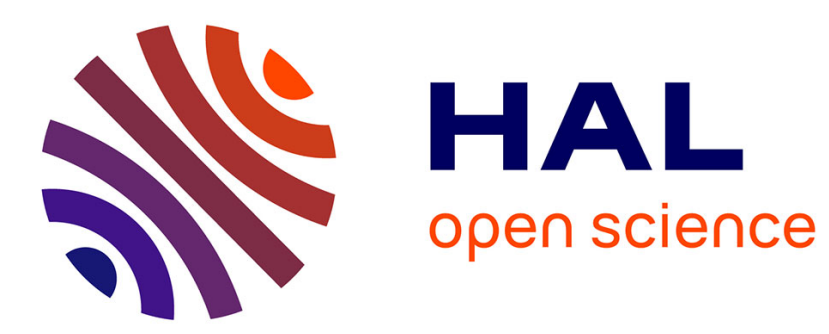

\title{
Three-dimensional motion computation and object segmentation in a long sequence of stereo frames
}

Zhengyou Zhang, Olivier Faugeras

\section{To cite this version:}

Zhengyou Zhang, Olivier Faugeras. Three-dimensional motion computation and object segmentation in a long sequence of stereo frames. [Research Report] RR-1438, INRIA. 1991. inria-00075122

\section{HAL Id: inria-00075122 \\ https://hal.inria.fr/inria-00075122}

Submitted on 24 May 2006

HAL is a multi-disciplinary open access archive for the deposit and dissemination of scientific research documents, whether they are published or not. The documents may come from teaching and research institutions in France or abroad, or from public or private research centers.
L'archive ouverte pluridisciplinaire HAL, est destinée au dépôt et à la diffusion de documents scientifiques de niveau recherche, publiés ou non, émanant des établissements d'enseignement et de recherche français ou étrangers, des laboratoires publics ou privés. 


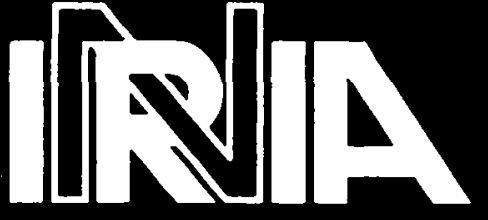

UNITÉ DE RECHERCHE NRIA-SOPHIA ANTIPOLIS

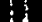

\section{Rapports de Recherche}

$$
\mathrm{N}^{\circ} 1438
$$

Programme 4

Robotique, Image et Vision

\section{THREE-DIMENSIONAL MOTION COMPUTATION AND OBJECT SEGMENTATION IN A LONG SEQUENCE OF STEREO FRAMES}

Institut National de Recherche en Informatique et en Automatique

Domaine de Voluceau Rocquencourt B.P.105

78153 Le Chesnay Cedex

France

Tél.:(1)39635511

Zhengyou ZHANG

Olivier D. FAUGERAS 
7 


\title{
Three-dimensional Motion Computation and Object Segmentation in a Long Sequence of Stereo Frames*
}

\author{
Estimation du mouvement $3 \mathrm{D}$ et \\ segmentation d'objets dans une séquence \\ longue de triplets stéréoscopiques
}

\author{
Zhengyou Zhang Olivier D. Faugeras \\ INRIA Sophia-Antipolis \\ 2004 route des Lucioles \\ 06565 Valbonne Cedex, France \\ zzhang@mirsa.inria.fr faugeras@mirsa.inria.fr
}

'This work was supported in part by Esprit projects P940 and Voila 



\begin{abstract}
We address the problem of computing the three-dimensional motions of objects in a long sequence of stereo frames. Our approach is bottom-up and consists of two levels. The first level deals with the tracking of $3 \mathrm{D}$ tokens from frame to frame and the estimation of their kinematics. The processing is completely parallel for each token. The second level groups tokens into objects based on their kinematic parameters, controls the processing at the low level to cope with problems such as occlusion, disappearance and appearance of tokens, and provides information to other components of the system. We have implemented this approach using 3D line segments obtained from stereo as the tokens. We use classical kinematics and derive closed-form solutions for some special, but useful, cases of motions. The motion computation problem is then formulated as a tracking problem in order to apply the extended Kalman filter. The tracking is performed in a prediction-matching-update loop in which multiple matches can be handled. Tokens are labeled by a number called its support of existence which measures their adequation to the measurements. If this number goes beyond a threshold, the token disappears. The individual line segments can be grouped into rigid objects according to the similarity of their kinematic parameters. Experiments using synthetic and real data have been carried out and the results found to be quite good.
\end{abstract}

Keywords: Image Sequence Analysis, 3D Motion Tracking and Computation, Kinematic Model, 3D Token tracker, Multiple Object Motions, Grouping, 3D Vision.

\title{
Résumé
}

Nous traitons le problème de l'estimation des mouvements d'objets dans une séquence longue de triplets stéréoscopiques. Notre approche est ascendante et est composée de deux niveaux. Au niveau inférieur, chaque attribut $3 \mathrm{D}$ est indépendamment suivi d'une vue à l'autre, et sa cinématique est estimée par un filtrage temporel. Le processus est complètement parallèle. Au niveau supérieur, nous groupons des attributs en objets selon la similarité des paramètres cinématiques. C'est l'intégration spatiale. Ce même niveau contrôle le processus du niveau inférieur pour résoudre les problèmes comme l'occlusion, la disparition et l'apparition. Nous avons implémenté cette approche en utilisant des segments $3 \mathrm{D}$ comme attributs reconstruits par un système stéréoscopique. Nous résolvons explicitement les equations de la cinématique pour ces attributs dans certains cas particuliers mais très utiles en pratique. Le problème de l'estimation du mouvement est formulé comme un problème de suivi auquel le filtre de Kalman étendu s'applique immédiatement. Le suivi est effectué dans une boucle de prédictionappariement-mise-à-jour et le problème des appariements multiples est traité. Les attributs sont caractérisés par un nombre, appelé son support d'existence, qui mesure leur adéquation aux observations. Si ce nombre dépasse un seuil, nous considérons que l'attribut a disparu. Nous avons mené des expériences en utilisant données synthétiques et données réelles. Les résultats sont présentés.

Mots clés: Analyse de séquences d'images, Estimation et suivi du mouvement 3D, Modèle cinématique, Suivi d'attributs 3D, Mouvements d'objets multiples, Groupement, Vision 3D. 



\section{Contents}

1 Introduction $\quad 3$

2 Statement of the Problem $\quad 6$

3 A Framework to Solve the Motion Tracking Problem $\quad 7$

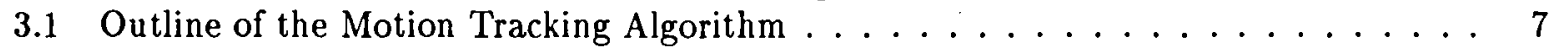

3.2 A pedagogical example . . . . . . . . . . . . . . . . . 9

4 Representation of 3D Line Segments 10

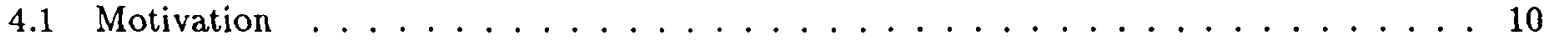

4.2 Our Representation . . . . . . . . . . . . . . . . . . 11

4.2.1 Representing the orientation by its Euler angles $\phi$ and $\theta \ldots \ldots \ldots \ldots 11$

4.2 .2 Modeling the midpoint of a 3D line segment . . . . . . . . . . . 12

5 Kinematic Model $\quad 13$

5.1 The Classical Kinematic Model . . . . . . . . . . . . . . . . . 13

5.2 Closed-form Solutions for Two Special Motions . . . . . . . . . . . . . . 15

6 Extended Kalman Filter $\quad 16$

7 Formulation of Motion Tracking Problem for EKF Approach 17

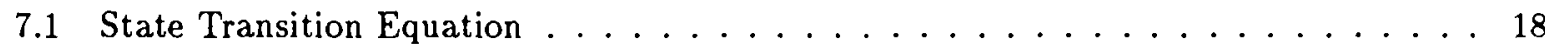

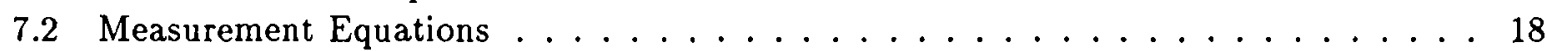

8 Matching Segments $\quad 20$

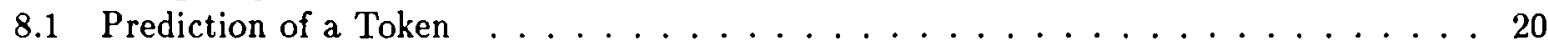

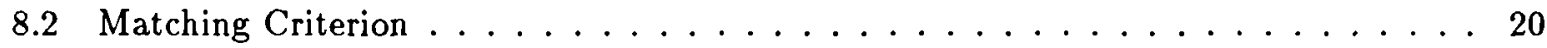

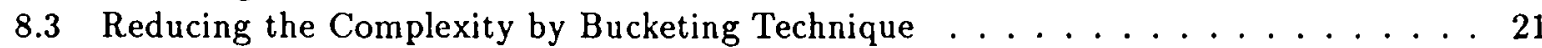

9 Support of Existence $\quad 22$

10 Grouping Tokens into Objects

11 Experimental Results $\quad 25$

11.1 Real Data with Controlled Motion . . . . . . . . . . . . . . 25

11.2 Real Data with Uncontrolled Motion . . . . . . . . . . . . . . . 32

12 Discussion $\quad 34$

13 Conclusion $\quad 37$

$\begin{array}{ll}\text { References } & 42\end{array}$ 


\section{List of Figures}

1 Illustration of the motion tracking problem $\ldots \ldots \ldots \ldots$

2 An Architecture for Motion Tracking . . . . . . . . . . . . . 7

3 Block Diagram of the Token Tracking Algorithm ............ 7

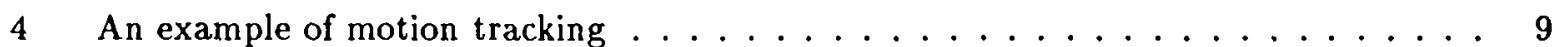

5 Illustration of the classical kinematics for rigid bodies $\ldots \ldots \ldots \ldots \ldots$

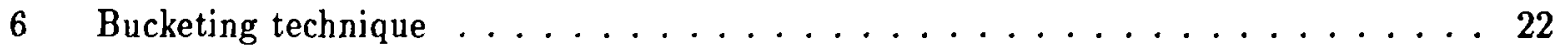

7 Description of the rotating table undergoing general motion $\ldots \ldots \ldots \ldots$

8 The images taken by the first camera at $t_{1}$ and $t_{10} \ldots \ldots \ldots \ldots \ldots$

9 The superposition of the first (in solid lines) and the second (in dashed lines) frames 28

10 The superposition of the first (in solid lines) and the last (in dashed lines) frames . . 29

11 The superposition of the predicted (in solid lines) and the observed (in dashed lines)

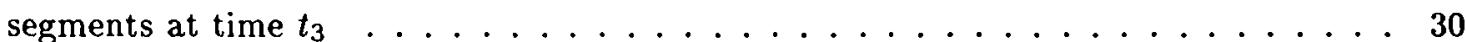

12 The superposition of the predicted (in solid lines) and the observed (in dashed lines)

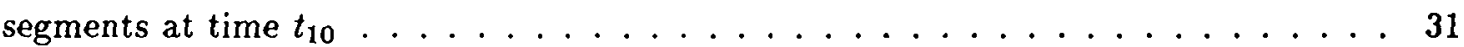

13 Error evolution in the angular velocity of the rotating table undergoing general motion 32

14 Error evolution in the translational velocity of the rotating table undergoing general

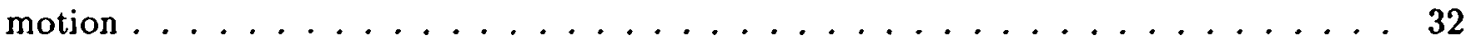

15 The images taken by the first camera at $t_{1}$ and $t_{15} \ldots \ldots \ldots \ldots \ldots$

16 A stereogram showing the superposition of the predicted (in solid lines) and observed (in dashed lines) segments at $t_{2} \ldots \ldots \ldots \ldots \ldots \ldots \ldots \ldots$

17 A stereogram showing the superposition of the predicted (in solid lines) and observed (in dashed lines) segments at $t_{3} \ldots \ldots \ldots \ldots \ldots \ldots \ldots \ldots$

18 A stereogram showing the superposition of the predicted (in solid lines) and observed

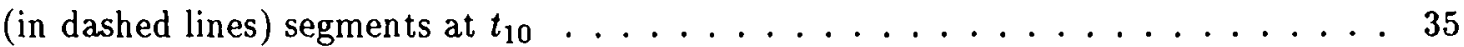

19 A stereogram showing the superposition of the predicted (in solid lines) and observed (in dashed lines) segments at $t_{15} \ldots \ldots \ldots \ldots \ldots \ldots \ldots$

20 Two different cases of multiple matches $\ldots \ldots \ldots \ldots \ldots$

21 An example to show the importance of merging $\ldots \ldots \ldots \ldots \ldots$ 


\section{Introduction}

The problem of analyzing sequences of images to extract three-dimensional motion and structure has been at the heart of the research in computer vision for many years. It is very important since its success or failure will determine whether or not vision can be used as a sensory process in reactive systems. There are of course many possibilities for attacking the problem and many more remain to be explored. We discuss a few of them.

In fact, image sequence analysis is a rather vague term and can cover several meanings. Our definition is that, given one or several sequences of images acquired from one or several cameras whose relative positions are known and which are rigidly moving in an unknown environment containing a number of mobile rigid objects, we must determine the various relative motions (cameras and objects) and the structure of the scene.

There has been a tremendous amount of work on the analysis of monocular sequences of images. This work has basically followed two main paths: optical flow and token tracking. The philosophy of optical flow is to work in two steps. First estimate from the variation of image intensities the projection of the three-dimensional velocities and second, compute those velocities (in fact the kinematic screw that defines them) and the depth from the optical flow. The reason for splitting the process of recovery into those two steps can be traced back to the work of Gibson (Gibson, 1950) and Koenderink (Koenderink and van Doorn, 1975; Koenderink and van Doorn, 1978; Koenderink, 1986). Because of fundamental difficulties such as the aperture effect, researchers have only been able to partially solve the first step (Horn and Schunk, 1981; Nagel, 1983; Nagel, 1986; Hildreth, 1984) and the results of the second step have been reported to be of very poor quality. These and other reasons such as the fact that the relationship between the optical flow and the projected velocity field is a bit uncertain (Faugeras, 1990) and the optical flow can only be reliably estimated near image discontinuities, have led researchers to explore another route, to track tokens.

The philosophy of the token tracking approach is also to work in two steps. First detect reliable tokens such as curves, corners, from the spatial variations of image intensities, assuming that they correspond to markings on the three-dimensional objects. Second, track them over time and recover the depth and three-dimensional velocities of the corresponding $3 \mathrm{D}$ tokens. This tracking is performed by building a kinematic model for the two-dimensional tokens. There are two main possibilities, either to work directly in $3 \mathrm{D}$ or to work in 2D. The advantage of the first option is that one works in the "right space" where it makes more sense to model the kinematics of objects but at the cost of being very sensitive to noise. The advantage of the second approach is that one works in the "easy space" where measurements are made but at the cost of not computing directly the values we are really interested in computing. This is where the largest amount of work has been performed (Sethi and Jain, 1987; Crowley et al., 1988; Gambotto, 1989; Hwang, 1989; Deriche and Faugeras, 1990;

Broida and Chellappa, 1986; Broida and Chellappa, 1989; Weng et al., 1987; Dickmanns, 1987; Dickmanns and Graefe, 1988b; Dickmanns and Graefe, 1988a).

Another possibility for performing this computation is to consider that the two-dimensional tracking gives us matches between different frames and estimate the three-dimensional motions from those matches. This last problem has also received considerable attention. A lot of work has been published on algorithms for recovering motion and structure from $n$ point matches, $p$ line matches, between $q$ views, where typically $n$ is $5, p$ is 6 , and $q$ is 2 or 3 (Ullman, 1979; Tsai and Huang, 1981; Huang and Tsai, 1981; Longuet-Higgins, 1981; Yen and Huang, 1983; Tsai and Huang, 1984; Zhuang and Haralick, 1985; Liu and Huang, 1986; Liu and Huang, 1988; Aggarwal and Wang, 1987; Faugeras and Maybank, 1990). These results are 
theoretically very interesting but are limited to the estimation of the motion of a single object and to the reconstruction of the structure of the scene up to a scale factor unless considerable a priori information is available. Also, due to the complexity of image formation and the nonlinear relation between 3D motion and changes in the images, the solutions have been reported to be very sensitive to noise, and thus have so far been of little practical use except, perhaps, for calibration.

There has also been a large amount of work on stereo (Baker and Binford, 1981; Grimson, 1981; Grimson, 1985; Nishihara, 1984; Ohta and Kanade, 1985; Pollard et al., 1985; Marr and Poggio, 1976; Marr and Poggio, 1979; Yachida, 1986; Ayache and Lustman, 1987; Kitamura and Yachida, 1990) which can be seen as the analysis of two or three sequences of images (if we use binocular or trinocular stereo), each sequence being limited to only one image. The main problem has been, and remains, to establish correspondences between the images and to reconstruct a depth map which is as dense as possible.

Much less has been done on the analysis of several, simultaneously acquired, sequences of images. Clearly the amount of information is much higher and one would hope that this would allow us to solve the problem in a more robust fashion. It is not obvious, however, to decide how to proceed and build upon existing techniques, for example those developed for the analysis of monocular sequences and stereo. In (Zhang et al., 1988; Zhang and Faugeras, 1991a; Zhang and Faugeras, 1991b), we have proposed an algorithm based upon the hypothesize-and-verify paradigm to match $3 \mathrm{D}$ line segments and to compute 3D displacements between two 3D frames obtained from stereo. In order to reduce the complexity of the method, we have made the assumption that objects are rigid. This algorithm has been extended to deal with the case where several mobile objects are present.

The solution which we explore in this paper is the following. We assume that we can do reliable stereo at a reasonable rate, let us say five times a second to fix ideas. We then match the set of sequentially reconstructed three-dimensional representations and estimate motion. Therefore, we do three-dimensional motion from three-dimensional structure.

Of course, there are many details that need to be filled in:

1. what are the three-dimensional representations that are used?

2. how do we match them?

3. how do we estimate motion from the matches?

The answer to point number 1 is that we use an edge-based stereo algorithm that has been developed in the past (Ayache and Lustman, 1987) and put into hardware (Faugeras et al., 1988b). It can deliver three-dimensional line segments at the rate of $5 \mathrm{~Hz}$. Therefore, our representation is quite simple and consists of sets of three-dimensional line segments. It is not clear how crucial this assumption is for the whole system. We believe that many of the ideas described in this paper can be used for other simple geometric primitives, other tokens, even though in the details of the current implementation the line segment assumption plays an important role.

The answer to points number 2 and 3 is that we build a model of the kinematics of each token, assuming that it is attached to a moving rigid object. We use this model to predict the appearance of the token in the three-dimensional visual map obtained at the next time instant. We use this prediction to verify whether the token is present and match it, if possible, to a real token. The match is then used to update the kinematic model. The whole process is implemented as a Kalman filter. Therefore, matching and estimation of motion are intimately related in this approach. One interesting feature is that we actually integrate the kinematic equations by making the assumption that the motion we observe can be well approximated on a short-time scale by constant angular 
velocity and constant linear acceleration. This is in general true only if the time sampling frequency is high enough so that the accelerations can be neglected between two sampling times.

We also tackle the following problems that arise when we deal with (long) sequences of images:

- Occlusion: A moving object may be partially or totally occluded by the background or by other objects.

- Disappearance: A moving object in the current field of view may move partially or totally out of it in the next frames.

- Appearance: A previously unseen object may partially or totally come into view.

Clearly, occlusion is related to disappearance and appearance, since when we talk about the occlusion of an object, we mean that some of its features disappear for a moment and may eventually reappear in the future. Those three events are due to regular transformations of the scene. We must add to them a fourth one which is due to the failure of the algorithms that produce the description:

- Absence: When features which should be present are not due to the failure of the feature extraction (or reconstruction) process.

These remarks bring forward an interesting aspect of the problem, namely that there are always two kinds of tokens: those which have been seen for a sufficiently long time so that the system has been able to build a good model of their kinematics, and those which have just entered the field of view and for which no kinematics information is available. The first kind of tokens is "easily" dealt with since it is likely that the prediction stage will help to cut down heavily the number of tentative candidates to a match in the next frame. For the second kind a computational explosion is likely to happen: in order to find the right match, we may have to explore a large number of possibilities and if we make the wrong choice we will lose track of the token. Therefore our system can be seen as operating in two modes, the first one called the continuous mode and the second called the bootstrapping mode.

The continuous mode applies to tokens for which the system has built up a kinematic model with low uncertainty. The model at time $t$ is used to predict the position and orientation of the token in the scene at time $t+\Delta t$. Since the uncertainty of the model is small, the search for corresponding tokens can be restricted to a small zone around the predicted token.

The bootstrapping mode assumes no knowledge of the kinematics of the token, i.e assumes that it is not moving, with a large uncertainty. Its position and orientation at time $t+\Delta t$ are predicted to be the same as those at time $t$ but, since the uncertainty of the model is large, the search for corresponding tokens is conducted in a larger zone than in the previous mode leading to the possibility of many candidates.

One interesting feature of both modes is that they use the idea of least-commitment and, instead of forcing a decision, may make multiple correspondence choices and use the time continuity to throw away later the ones which are not confirmed by the measurements.

Another feature of our approach is that we can detect multiple motions by grouping tokens which have similar kinematic models, thus obtaining a segmentation of the scene into "objects" (i.e sets of tokens) moving rigidly.

We are not the first ones to investigate this problem from that viewpoint. Young and Chellappa (Young and Chellappa, 1988) describe the computer simulation of a system that uses a number of noisy $3 \mathrm{D}$ points assumed to belong to the same rigid object to estimate its motion. In their work, the problem of obtaining the matches from frame to frame and the problem of multiple objects are not addressed. 

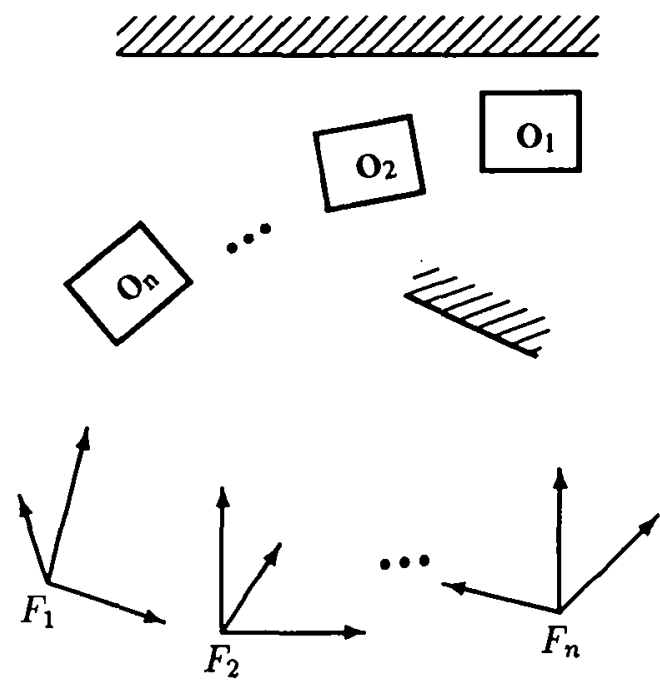

Fig. 1: Illustration of the motion tracking problem

\section{Statement of the Problem}

We address the motion tracking problem that arises in the context of a mobile vehicle navigating in an unknown environment where other mobile bodies such as human beings or robots may also be moving. A stereo rig mounted on the mobile vehicle provides a sequence of $3 \mathrm{D}$ maps of the environment. The current stereo system is trinocular (Ayache and Lustman, 1987), and the 3D tokens we are using are line segments produced by significant intensity discontinuities in the images. Although the framework to solve the motion tracking problem developed in this paper arises in this specific context, we believe it should be applicable in other contexts; in particular we could use other $3 \mathrm{D}$ primitives, for example, points, combinations of points and lines, curves.

The situation is illustrated in Figure 1. The static environment is represented by cross-hatched regions. Only one moving object is drawn, represented by a square. The mobile robot is represented by a frame of reference, which is the one of the stereo system. This reference frame is attached to the mobile robot and its numerical parameters are determined in the camera calibration phase (Faugeras and Toscani, 1986). In the figure, the object undergoes a general motion from right to left, and the robot moves from left to right. We want to solve the following problems:

1. Find the positions of static and moving objects in each stereo frame,

2. Determine the motion of the robot as well as those of the moving objects with respect to the static environment.

As stated earlier, in order to resolve these issues we have to handle problems in dynamic scene analysis such as occlusion, appearance, disappearance, and absence of features.

Those problems can be solved at the level of ob jects: object tracking, or at the level of features which constitute objects: token tracking. In the object tracking approach, the scene must first be segmented in to objects, which in general requires high-level knowledge about the characteristics of objects such as rigidity and geometry (planar world). This approach is in general difficult. In some special cases, such as Radar imagery and in the experiment reported in (Gordon, 1989) using 
tennis balls, objects can be easily detected and can be replaced by points (usually their centers of gravity). In the token tracking approach, no such knowledge is required and the tracking process can be carried out in parallel for each token. For this reason and also for the followings, we track $3 \mathrm{D}$ line segments instead of $3 \mathrm{D}$ objects:

- Objects can be later identified by grouping line segments with similar motion,

- After tracking individual line segments, one can detect multiple moving objects, articulated objects or even deformable objects based on common motion characteristics.

Because of this, the hypothesis which assumes that objects are moving rigidly can be somewhat relaxed in the analysis of long sequences.

\section{A Framework to Solve the Motion Tracking Problem}

To clarify the presentation, we call the 3D line segments being tracked the tokens and the currently observed $3 \mathrm{D}$ line segments the scene tokens.

\subsection{Outline of the Motion Tracking Algorithm}

Our motion tracking algorithm consists of two levels (see Figure 2). In the figure, single arrows represent data flow, and the double arrow represents the flow of control.

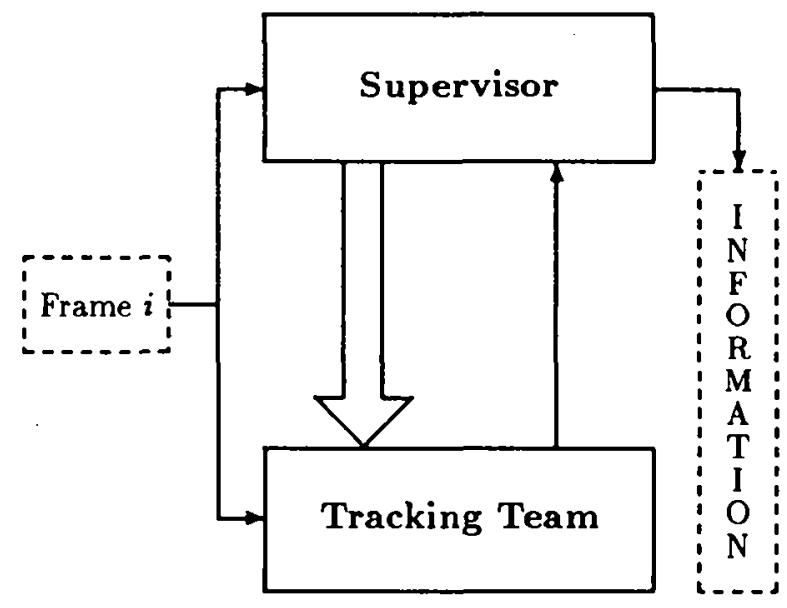

Fig. 2: An Architecture for Motion Tracking

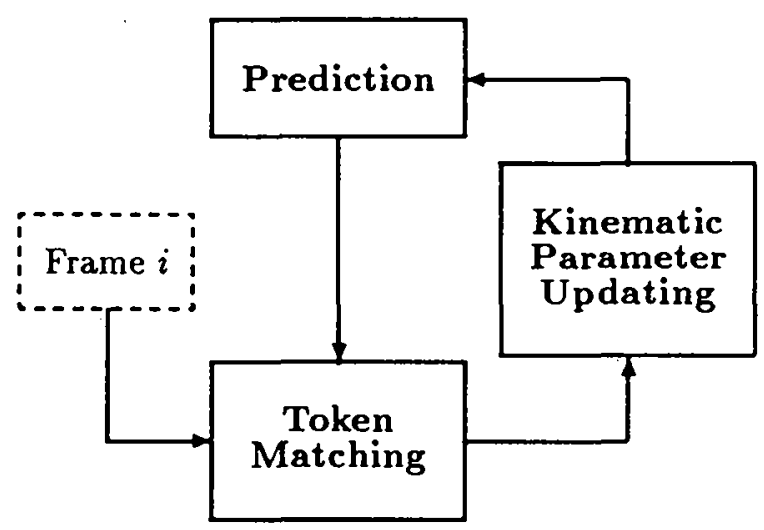

Fig. 3:
Block Diagram of the Token Tracking Algorithm

The low level is called the tracking team. A token being tracked can be considered as one of the team members. As we discussed earlier, the token tracking process uses a model of the $3 \mathrm{D}$ kinematics, instead of a model of the evolution of the token parameters. A token is then characterized by its position and orientation in the current frame, its kinematic parameters, and a positive number called support. The support indicates the degree of support for the existence of the token, which will be described in section 9.

When a new frame is acquired, each token being tracked "searches" the whole frame for a correspondence. The search space can be considerably reduced by using a kinematic model and information from previous frames: before the new frame is available, one can predict the occurrence 
of each token in the new frame based on the kinematic model. When the new frame is obtained, one only needs to look for scene tokens in the neighborhood of the predicted position.

When a match is found, the parameters of the token kinematic model are updated, the position parameters are replaced by those of its match*, and the support parameter is also updated. The prediction and update of the position and kinematic parameters are done by using an Extended Kalman Filter. The matching process is based on the Mahalanobis distance. Due to occlusion or absence of some scene tokens in the current frame, a token may not find any match in the neighborhood of its predicted position in the current frame. Of course, this phenomenon may also occur due to the disappearance of the token. To handle the occlusion and absence of scene tokens, it is necessary to hypothesize the existence of the token and continue to change its kinematic parameters according to the kinematic model and update its support.

Figure 3 shows the block diagram of the algorithm, and the details will be described in the following sections. As we can observe, the above process can be performed independently for each token to be tracked, and this allows a completely parallel implementation.

The high level is called the supervisor. It has three main functions:

- Grouping tokens with similar kinematic parameters as a single object. If there exist multiple moving objects, they can be segmented on the grounds that they undergo different motions. We describe later the details about how to group tokens.

- Monitoring the tracking team by detecting the following events:

1. Appearances: When a new token appears, i.e., when a scene token in the current frame cannot be matched with any token being tracked, then the supervisor activates an additional token in the tracking team. This new token starts the same process as the others.

2. False matches: When a token loses the support of its existence (see Section 9), the supervisor then deactivates this token. Usually such tokens have been activated due to false matching in the previous frames. In a parallel implementation, the processor occupied by this token would be freed, and could be used by some new token.

3. Disappearances: When a token moves out of the field of view, the supervisor deactivates this token. We can easily determine whether a tracked token is out of the field of view by projecting it onto one of the camera planes. Just as in the previous case, in a parallel implementation, the processor occupied by this token would be freed, and could be reutilized.

4. Multiple matches: A token being tracked may find multiple matches in the current frame with a criterion defined $a$ priori (the Mahalanobis distance, for example), especially when there are several scene tokens which are near to each other. A common way to solve this problem is to choose the scene token which is the nearest to the predicted position (best-first search), as in (Crowley et al., 1988; Deriche and Faugeras, 1990). This may lead to unpredictable results. A more robust approach is to keep tracking the token using several nearest scene tokens in the current frame; thus a token can be split. This approach can be called beam search. In our implementation, we choose the two nearest scene tokens to the predicted position in the sense of the Mahalanobis distance (see

\footnotetext{
- One can also update the position parameters by modifying a little the state vector in the formulation of Section 7 . The computation will be more expensive, as the complexity of EKF is $O\left(n^{3}\right)$, where $n$ is the dimension of the state vector.
} 
Section 8), if their distances are both less than some threshold. The token updates its kinematic parameters by incorporating the nearest scene token. If the second nearest scene token exists, then the token reports it to the supervisor. The supervisor activates an additional token by integrating the original token and the matched one. The beam search strategy is utilized in other research fields, such as in the HARPY speech understanding system (Lowerre and Reddy, 1980). This strategy has been found to be efficient as well as robust.

5. About changes: A potential capacity of the supervisor to monitor the tracking team is to detect abrupt changes in the motion of a token due, for example, to collision, and to reinitialize its kinematic parameters.

- Providing information to other components of the global system. For example, in an active tracking application, one may need to control the motion of the robot or adjust the camera parameters to adapt the changing situation based on the information provided by the motion tracking algorithm. The information may include the kinematics of the robot (ego-motion) and the kinematics and relative positions of the moving objects.

\subsection{A pedagogical example}

Figure 4 shows an example of how the tracking team works. At $t_{2}$, token 1 is split in two (token 1 and token $1^{\prime}$ ) due to ambiguous matches. At $t_{3}$, token $1^{\prime}$ cannot find a correspondence in the current frame, and it makes an hypothetical extension to cope with the occlusion problem. But because too many such hypothetical extensions are made consecutively, it loses its support for existence at $t_{5}$ and is then deactivated. At $t_{5}$, token 1 cannot find its correspondence in the current frame, and it makes an hypothetical extension. It finds its correspondence at $t_{6}$. Thus the occlusion problem is handled gracefully.

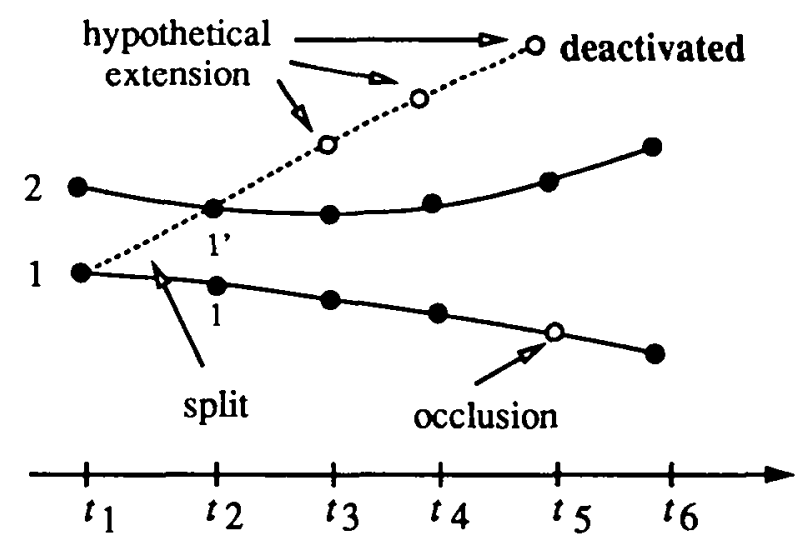

Fig. 4: An example of motion tracking 


\section{Representation of 3D Line Segments}

Our stereo system reconstructs a set of 3D line segments about its environment. These segments may correspond to the contours of objects, to shadows or to region markings. Line segments addressed here are oriented thanks to the intensity contrast. In this section, we propose a new representation for a line segment which we think is well adapted for the task at hand.

\subsection{Motivation}

It is a consensus among many researchers in the field that uncertainty should be explicitly represented and manipulated in computer vision and robotics applications (Ayache and Faugeras, 1989; Durrant-Whyte, 1988). We can think of uncertainty as follows. Let us model the features as random and consider their probability density functions. In practice those functions are very hard to estimate and one is usually satisfied with the first few moments, usually the first two, the mean vector and the covariance matrix. This does not imply that the features are modeled as Gaussian but only that we neglect their higher order moments. The question of whether this poses problems, for example with the Kalman filter, is answered in Section 6.

A related question is the following. Suppose that we have a random feature vector $\mathbf{x}$ with mean $x_{0}$ and covariance matrix $\Lambda_{\mathbf{x}}$ to which we apply a nonlinear function $f$ to produce a new random feature $y$. The question is to compute the mean and covariance matrix of $y$. One way to do this is to compute the Taylor series expansion of $\mathbf{f}$ in the vicinity of $\mathbf{x}_{0}$. If we perform this expansion up to the second order, we obtain:

$$
\mathbf{y}=\mathbf{f}(\mathbf{x})=\mathbf{f}\left(\mathbf{x}_{0}\right)+\mathbf{f}^{\prime}\left(\mathbf{x}_{0}\right)\left(\mathbf{x}-\mathbf{x}_{0}\right)+O\left(\left\|\mathbf{x}-\mathbf{x}_{0}\right\|^{2}\right)
$$

This shows that, up to the first order, we have:

$$
E(\mathbf{y})=\mathbf{f}\left(\mathbf{x}_{0}\right)=\mathbf{f}(E(\mathbf{x}))
$$

and

$$
\Lambda_{\mathbf{y}}=E\left[(\mathbf{y}-E(\mathbf{y}))(\mathbf{y}-E(\mathbf{y}))^{T}\right]=\mathbf{f}^{\prime}\left(\mathbf{x}_{0}\right) \Lambda_{\mathbf{x}} \mathbf{f}^{\prime}\left(\mathrm{x}_{0}\right)^{T}
$$

Of course these may be poor approximations if the second order term is not negligible. For example, up to the third order, the mean is given $\mathrm{by}^{\dagger}$ :

$$
E(y)=f\left(x_{0}\right)+\frac{1}{2} f^{\prime \prime}\left(x_{0}\right) \sigma_{x}^{2}
$$

This formula clearly shows that the fact that the second order term can or cannot be neglected depends upon both the magnitude of the second order derivative of $f$ and of the variance of $x$.

In this paper, we assume that the first order approximation is sufficient either because the second order derivatives are small compared to the first order derivatives, or because the second order moments are small, or both.

A line segment is usually represented by its endpoints $M_{1}$ and $M_{2}$, which require 6 parameters, and their covariance matrices $\Lambda_{1}$ and $\Lambda_{2} . \Lambda_{1}$ and $\Lambda_{2}$ are estimated by stereo triangulation from point correspondences (Ayache, 1988). Equivalently, a line segment can be represented by its direction vector $\mathrm{v}$, its length $l$, and its midpoint $M$, and their covariance matrices.

\footnotetext{
${ }^{\dagger}$ For simplicity, we assume that $x$ and $y$ are scalar.
} 
But we cannot directly use these parameters in most cases. As explained in section 4.2 .2 , the endpoints or the midpoint of a segment are not reliable. Thus, instead of the line segment, the infinite line supporting the segment is usually used, as in (Kim and Aggarwal, 1987). In an earlier version of our algorithm for motion analysis of two stereo views (Ayache and Faugeras, 1987a; Faugeras et al., 1988a; Zhang et al., 1988), a line segment is treated in a mixed way. The infinite supporting line is used in estimating motion and the line segment is used in matching.

Many representations have been proposed in the literature for a line segment (Ayache and Faugeras, 1987b; Roberts, 1988). The main problem is that the uncertainty on the line parameterization does not reflect that of the segment which the line supports (see (Zhang, 1990) for more details). A segment with big uncertainty may yield a small uncertainty in the line parametrization, for example, if its uncertainty is in the direction of the line.

\subsection{Our Representation}

Because of the deficiencies of the previous representations for a line or a line segment, we use a five parameter representation for a line segment: two for the orientation, three for the position of a segment. This is a trade-off between an infinite line and a line segment. If we add the length, a line segment can then be fully specified. Special attention is given to the representation of uncertainty.

\subsubsection{Representing the orientation by its Euler angles $\phi$ and $\theta$}

Let us consider the spherical coordinates. Let $\mathbf{u}=\left[u_{x}, u_{y}, u_{z}\right]^{t}$ be a unit vector of orientation, we have:

$$
\left\{\begin{array}{l}
u_{x}=\cos \phi \sin \theta \\
u_{y}=\sin \phi \sin \theta \\
u_{z}=\cos \theta
\end{array}\right.
$$

with $0 \leq \phi<2 \pi, 0 \leq \theta \leq \pi$.

From $u$, we can compute $\phi, \theta$ :

$$
\begin{aligned}
& \phi= \begin{cases}\arccos \frac{u_{x}}{\sqrt{1-u_{x}^{2}}} & \text { if } u_{y} \geq 0 \\
2 \pi-\arccos \frac{u_{x}}{\sqrt{1-u_{z}^{2}}} & \text { otherwise }\end{cases} \\
& \theta=\arccos u_{z} .
\end{aligned}
$$

If we denote $[\phi, \theta]^{t}$ by $\psi$, then the mapping between $\psi$ and $\mathbf{u}$ is 1 -to-1, except when $\theta=0$. When $\theta=0, \phi$ is not defined. This will show in the covariance matrix of $\psi$ as a very large entry for the variance of $\phi$, indicating that the $\phi$-measurement cannot be trusted very much (Zhang, 1990).

Another problem with this representation is the discontinuity in $\phi$ when a segment is nearly parallel to the plane $y=0$. In that case, the angle $\phi$ may jump from the interval $[0, \pi / 2)$ to the interval $(3 \pi / 2,2 \pi)$, or vice versa. This discontinuity must be dealt with in matching and integration.

In the following, we assume that the direction vector $\mathrm{v}=[x, y, z]^{t}$ and its covariance matrix $\Lambda_{\mathbf{V}}$ of a given segment are known. We want to compute $\psi$ and its covariance matrix $\Lambda_{\psi}$ from $\mathbf{v}$ and $\Lambda_{\mathbf{V}} . \phi$ and $\theta$ are simply given by:

$$
\begin{aligned}
& \phi= \begin{cases}\arccos \frac{x}{\sqrt{x^{2}+y^{2}}} & \text { if } y \geq 0 \\
2 \pi-\arccos \frac{x}{\sqrt{x^{2}+y^{2}}} & \text { otherwise }\end{cases} \\
& \theta=\arccos \frac{z}{\sqrt{x^{2}+y^{2}+z^{2}}} .
\end{aligned}
$$


Since the relation between $\psi$ and $\mathbf{v}$ is not linear, we use the first order approximation of equation 1 to compute the covariance matrix $\Lambda_{\psi}$ from $\Lambda_{v}$. That is

$$
\Lambda_{\psi}=\frac{\partial \psi}{\partial \mathbf{v}} \Lambda_{\mathbf{v}} \frac{\partial \psi^{t}}{\partial \mathbf{v}}
$$

where the Jacobian matrix $\frac{\partial \psi}{\partial v}=\left[\begin{array}{lll}\frac{\partial \phi}{\partial x} & \frac{\partial \phi}{\partial y} & \frac{\partial \phi}{\partial z} \\ \frac{\partial \theta}{\partial x} & \frac{\partial \theta}{\partial y} & \frac{\partial \theta}{\partial z}\end{array}\right]$.

\subsubsection{Modeling the midpoint of a $3 \mathrm{D}$ line segment}

We choose the midpoint as the three parameters to localize a segment, but a special treatment on the covariance is introduced to characterize the uncertainty in the location of a segment.

The reason for this is that the way the uncertainty of the endpoints of a three-dimensional segment is computed takes only into account the uncertainty of the pixel coordinates due to the edge detection process and the uncertainty of the calibration of the stereo rig (Ayache and Faugeras, 1989). But it does not take into account the uncertainty due to the variations in the different images of the stereo triplet of the polygonal approximations of corresponding contours. There are two main sources for these variations.

The first is purely algorithmic: because of noise in the images and because we sometimes approximate significantly curved contours with line segments, the polygonal approximation may vary from frame to frame inducing a variation in the segments endpoints which has not been accounted for. The second is physical: because of partial occlusion in the scene, a segment can be considerably shortened or lengthened and this has also to be taken into account in the modeling of uncertainty.

In an attempt to cope with all this, we model the midpoint $m$ of a segment $M_{1} M_{2}$ as:

$$
\mathbf{m}=\mathbf{M}+n \mathbf{u},
$$

where $\mathrm{M}=\left(M_{1}+M_{2}\right) / 2, \mathbf{u}$ is the unit direction vector of the segment and $n$ is a random scalar. Equation 6 says in fact that the midpoint has some extra uncertainty attached to it. It may vary randomly along the line supporting it in successive views.

The random variable $n$ in Equation 6 is modeled as zero-mean with deviation $\sigma_{n}$, a positive scalar. If a segment is reliable, $\sigma_{n}$ may be chosen to be a small number; if not, it may be chosen to be a big number. In our implementation, $\sigma_{n}$ is related to the length $l$ of the segment, i.e., $\sigma_{n}=\kappa l$, where $\kappa$ is some constant. That is to say that the longer a segment is, the bigger the deviation $\sigma_{n}$ is. That is reasonable since a long segment is much likely to be broken into smaller segments in other views. In our experiments, $\kappa=0.2$.

In order to compute the covariance of $\mathbf{m}$, we should first compute $\mathbf{u}$ and $\Lambda_{\mathbf{u}}$. The unit direction vector $\mathbf{u}$ and its covariance $\Lambda_{\mathbf{u}}$ can be computed from a non-normalized direction vector $\mathbf{v}$ and its covariance matrix $\Lambda_{v}$ from equation 1 . Indeed, we have

$$
\mathbf{u}=\frac{\mathbf{v}}{\|\mathbf{v}\|}, \quad \Lambda_{\mathbf{u}}=\frac{\partial \mathbf{u}}{\partial \mathbf{v}} \Lambda_{\mathbf{v}} \frac{\partial \mathbf{u}^{t}}{\partial \mathbf{v}}
$$

where $\frac{\partial u}{\partial \mathbf{v}}$ is a $3 \times 3$ matrix $\left(\frac{\partial \mathbf{u}}{\partial \mathbf{v}}=\frac{\mathbf{I}_{3}}{\|\mathbf{v}\|}-\frac{\mathbf{v v} \mathbf{t}}{\|\mathbf{v}\|^{3}}\right)$. Note that the covariance matrix $\Lambda_{\mathbf{u}}$ is singular (the determinant is zero). This is due to the fact that the three components of $\mathbf{u}$ are not independent since $\|\mathbf{u}\|=1$. 
At this point, the covariance of $\mathbf{m}$ can be computed. We start with the covariance of $n \mathbf{u}$. Since $n$ and $u$ are independent of each other, we have

$$
\begin{gathered}
\mathrm{E}[n \mathbf{u}]=\mathrm{E}[n] \mathrm{E}[\mathbf{u}]=0, \\
\Lambda_{n \mathbf{u}}=\mathrm{E}\left[(n \mathbf{u})(n \mathbf{u})^{t}\right]=\mathrm{E}\left[n^{2}\right] \mathrm{E}\left[\mathbf{u} \mathbf{u}^{t}\right]=\sigma_{n}^{2}\left(\Lambda_{\mathbf{u}}+\mathbf{u} \mathbf{u}^{t}\right),
\end{gathered}
$$

where $\mathbf{a}=E[\mathbf{u}]$. Now we have:

$$
\mathrm{E}[\mathbf{m}]=\mathrm{E}[M]+\mathrm{E}[n \mathbf{u}]=\mathrm{E}[M]
$$

and:

$\Lambda_{\mathbf{m}}=\mathrm{E}\left[(\mathbf{m}-\mathrm{E}[\mathbf{m}])(\mathbf{m}-\mathrm{E}[\mathbf{m}])^{t}\right]=\mathrm{E}\left[(\mathrm{M}-\mathrm{E}[\mathrm{M}])(\mathrm{M}-\mathrm{E}[\mathrm{M}])^{t}\right]+\mathrm{E}\left[(n \mathbf{u})(n \mathbf{u})^{t}\right]+\mathrm{E}\left[n(\mathrm{M}-\mathrm{E}[\mathrm{M}]) \mathbf{u}^{t}\right]$

Since $n$ is independent of $\mathbf{M}$ and $\mathbf{u}$ and has zero-mean, the last two terms are equal to 0 and

$$
\Lambda_{\mathbf{m}}=\Lambda_{M}+\Lambda_{n \mathbf{u}}
$$

We consider that $M_{1}$ and $M_{2}$ are independent, therefore

$$
\Lambda_{M}=\left(\Lambda_{1}+\Lambda_{2}\right) / 4
$$

and this completes the computation of $\Lambda_{M}$, up to the first order.

If we add another parameter $l$ to denote the length of the segment, we can then represent exactly a line segment. This ends our modeling of a line segment. See (Zhang and Faugeras, 1990a; Zhang, 1990) for more details.

\section{$5 \quad$ Kinematic Model}

A common approach to model the motion kinematics is to divide the motion into two parts: a rotation about a point (called the center of rotation) and a translation of the center of rotation. The rotation is often assumed to be constant angular velocity or constant precession. The trajectory of the rotation center is assumed to be well approximated by the first $k$ terms of a polynomial $(k \geq 0)$. See (Broida and Chellappa, 1986; Broida and Chellappa, 1989; Weng et al., 1987; Young and Chellappa, 1988) for such a modelling. We show in (Zhang, 1990) that in case of constant angular velocity, that modelling is a special case of the one described in this section. In (Webb and Aggarwal, 1982), the fixed axis assumption is used to recover the 3D structure of moving rigid and jointed objects from several single camera views. The fixed axis assumption is stated as follows: Every rigid object movement consists of a translation plus a rotation about an axis that is fixed in direction for short periods of time. In this section, we describe the well-known model of classical rigid bodies kinematics and then derive the closed-form solutions for some special motions.

\subsection{The Classical Kinematic Model}

Given a Cartesian system of reference $O x y z$ for rigid bodies in which a rigid body is in motion. Choose a point on the solid, noted by $P$. Consider any point $M$ of the solid, then its velocity $\mathbf{v}_{M}$ is the sum of the velocity $v_{P}$ of the point $P$ and the rotation around the point $P$ (see Figure 5), that is

$$
\mathbf{v}_{M}(t)=\mathbf{v}_{P}(t)+\omega(t) \times \overrightarrow{P M}
$$




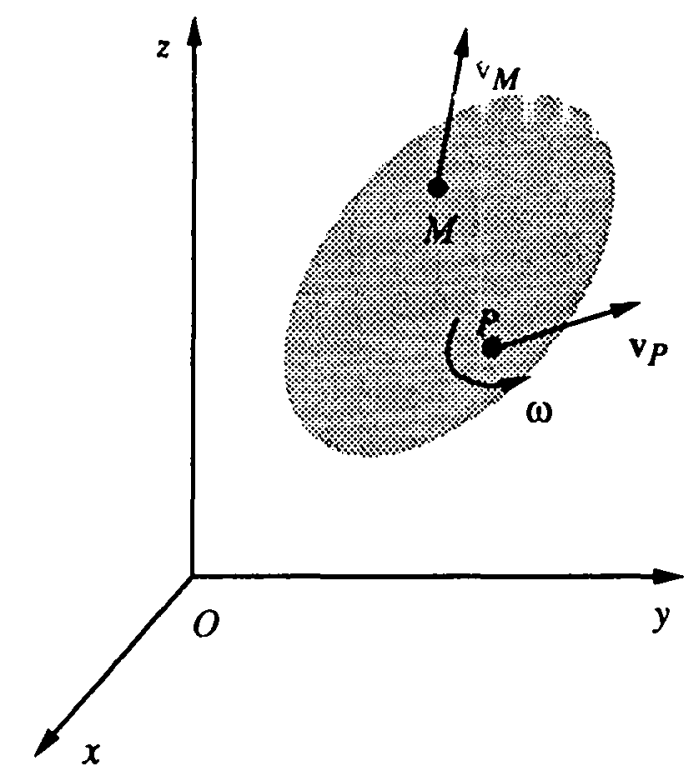

Fig. 5: Illustration of the classical kinematics for rigid bodies

where $\omega(t)$ is called the angular velocity, and $x$ denotes the cross product of two vectors.

The above equation is true for any point $P$. For simplicity, we choose the origin as the point $P$, i.e., $P=O$, and we have the kinematic model as following:

$$
\mathbf{v}_{M}(t)=\mathbf{v}(t)+\omega(t) \times \overrightarrow{O M} .
$$

The kinematics of any point $M$ of the body is completely characterized by $\mathbf{v}(t)$, the velocity of the point of the solid coinciding with the origin of the reference system, and $\boldsymbol{\omega}(t)$, the angular velocity of the point $M$ around the origin. The pair $(\omega(t), \mathbf{v}(t))$ is called the kinematic screw of the solid.

Let us replace $\overrightarrow{O M}$ in Equation 11 by $\mathbf{p}(t)$, and $\mathbf{v}_{M}(t)$ by $\dot{\mathbf{p}}(t)$, where $\dot{\mathbf{p}}(t)$ denotes the time derivative of $\mathbf{p}(t)$, i.e., $\frac{d \mathbf{p}(t)}{d t}$. For the sake of clarity, we write the time as a subscript. For instance, $\mathbf{p}(t)$ is written as $\mathbf{p}_{t}$. If we denote by $\tilde{\mathbf{v}}$ the antisymmetric matrix associated with $\mathbf{v}=\left[v_{1}, v_{2}, v_{3}\right]^{t}$, i.e.,

$$
\widetilde{\mathrm{v}}=\left[\begin{array}{ccc}
0 & -v_{3} & v_{2} \\
v_{3} & 0 & -v_{1} \\
-v_{2} & v_{1} & 0
\end{array}\right],
$$

then we have $\mathbf{v} \times \mathbf{u}=\widetilde{\mathbf{v}} \mathbf{u}$. Equation 11 can therefore be rewritten as a first order differential equation in $\mathbf{p}_{t}$ :

$$
\dot{\mathbf{p}}_{t}=\widetilde{\omega}_{t} \mathbf{p}_{t}+\mathbf{v}_{t}
$$

It is very difficult to get the solution of Equation 13 for a general motion. In the appendix of this paper, we show that a closed form can be obtained if the angular velocity is constant and the translational velocity is described by a polynomial of degree $n(n \geq 0)$. We give in the following section the closed form of the kinematic models for two special motions. 


\subsection{Closed-form Solutions for Two Special Motions}

In the case of constant angular and translational velocities, we have a simple closed-form of the solution (Theorem 1). Let $\boldsymbol{\omega}_{t}=\boldsymbol{\omega}$ and $\mathbf{v}_{t}=\mathbf{v}$.

Theorem 1: Trajectory in the case of constant angular and translational velocities

The trajectory of a point $\mathbf{p}_{t}$ given by Equation 13 is given, in the case of constant angular velocity $\omega$ and translational velocity $\mathbf{v}$, as:

$$
\mathbf{p}_{t}=W \mathbf{p}_{0}+V \mathbf{v}
$$

where

$$
\begin{aligned}
& W=\mathbf{I}_{3}+\frac{\sin (\theta \Delta t)}{\theta} \tilde{\boldsymbol{\omega}}+\frac{1-\cos (\theta \Delta t)}{\theta^{2}} \tilde{\boldsymbol{\omega}}^{2}, \\
& V=\mathbf{I}_{3} \Delta t+\frac{1-\cos (\theta \Delta t)}{\theta^{2}} \tilde{\boldsymbol{\omega}}+\frac{\theta \Delta t-\sin (\theta \Delta t)}{\theta^{3}} \widetilde{\boldsymbol{\omega}}^{2},
\end{aligned}
$$

and $\theta=\|\omega\|, \Delta t=t-t_{0}, \mathbf{I}_{3}$ is the $3 \times 3$ identity matrix, and $\mathbf{p}_{0}=\mathbf{p}_{t_{0}}$.

See the appendix for the proof. From Theorem 1, we can observe that when $\boldsymbol{\omega}=\mathbf{0}$ (i.e., pure translation), then

$$
\mathbf{p}_{t}=\mathbf{p}_{0}+\mathbf{v}\left(t-t_{0}\right)
$$

This is the well-known equation for a point moving on a straight line with constant velocity.

When angular velocity and translational acceleration are constant, we have the following equations:

$$
\begin{aligned}
& \boldsymbol{\omega}_{t}=\boldsymbol{\omega}, \\
& \mathbf{v}_{\boldsymbol{t}}=\mathbf{v}+\mathbf{a}\left(t-t_{0}\right),
\end{aligned}
$$

where $\boldsymbol{\omega}$ denotes the constant angular velocity, $\mathbf{v}$ denotes the translational velocity at $t=t_{0}$, and a denotes the constant translational acceleration. The trajectory of a point in this case is defined by the following theorem:

Theorem 2: Trajectory with constant angular velocity and translational acceleration

The trajectory of a point $\mathbf{p}_{t}$ given by Equation 13 is given, in the case of constant angular velocity $\omega$ and constant translational acceleration a, as:

$$
\mathbf{p}_{t}=W \mathbf{p}_{0}+V \mathbf{v}+A \mathbf{a},
$$

where $W$ is the same as in Equation 15, $V$ is the same as in Equation 16, and

$$
A=\frac{\Delta t^{2}}{2} \mathbf{I}_{3}+\frac{\theta \Delta t-\sin (\theta \Delta t)}{\theta^{3}} \tilde{\omega}+\frac{(\theta \Delta t)^{2}-2(1-\cos (\theta \Delta t))}{2 \theta^{4}} \tilde{\omega}^{2},
$$

and $\theta=\|\omega\|, \Delta t=t-t_{0}$ and $\mathbf{I}_{3}$ is the $3 \times 3$ identity matrix.

See the appendix for the proof. From Theorem 2, we observe that when $\omega=0$ (i.e., pure translation), then

$$
\mathbf{p}_{t}=\mathbf{p}_{0}+\mathbf{v}\left(t-t_{0}\right)+\mathbf{a} \frac{\left(t-t_{0}\right)^{2}}{2} .
$$

This is the well-known equation for a point moving on a straight line with constant acceleration. 


\section{Extended Kalman Filter}

In this section, we adapt the Extended Kalman Filter (EKF) (Maybeck, 1982) formulation to our problem. It will then be used in the next section to solve our motion tracking problem. For more details, the reader is referred to (Maybeck, 1979; Maybeck, 1982).

In practice, the individual state variables of a dynamic system cannot be determined exactly by direct measurements; instead, we usually find that the measurements that we make are functions of the state variables and that these measurements are corrupted by random noise. The system itself may also be subjected to random disturbances. We want to estimate the state variables from the noisy observations.

If we denote the state vector by $\mathbf{s}$ and denote the measurement vector by $\mathbf{x}$, a dynamic system (in discrete-time form) can be described by

$$
\begin{array}{cc}
\mathbf{s}_{i+1}=\mathbf{h}_{i}\left(\mathbf{s}_{i}\right)+\mathbf{n}_{i}, & i=0,1, \cdots, \\
\mathbf{f}_{i}\left(\mathbf{x}_{i}, \mathbf{s}_{i}\right)=0, & i=0,1, \cdots .
\end{array}
$$

Equation 22 is the state equation, and Equation 23 the measurement equation. In Equation $22, \mathbf{n}_{i}$ is the vector of random disturbance of the dynamic system and is usually modeled as white noise:

$$
E\left[\mathbf{n}_{i}\right]=0 \text { and } E\left[\mathbf{n}_{\mathbf{i}} \mathbf{n}_{j}^{t}\right]=\delta_{i j} Q_{i} .
$$

Where $\delta_{i j}=1$ if $i=j$ and 0 otherwise is the Kronecker symbol. The measurement $\mathbf{x}_{i}$ is corrupted by additive random noise, that is

$$
E\left[\mathbf{x}_{i}\right]=\overline{\mathbf{x}}_{i} \text { and } E\left[\left(\mathbf{x}_{i}-\overline{\mathbf{x}}_{\mathbf{i}}\right)\left(\mathbf{x}_{i}-\overline{\mathbf{x}}_{i}\right)^{t}\right]=\Lambda_{\mathbf{x}_{i}} .
$$

We assume also that there is no correlation between the noise process of the system and that of the observation, that is

$$
E\left[\left(\mathbf{x}_{i}-\overline{\mathbf{x}}_{i}\right) \mathbf{n}_{i}^{t}\right]=0 .
$$

When $\mathbf{h}_{i}$ and $\mathbf{f}_{i}$ are linear functions, we write $\mathbf{s}_{i+1}=H_{i} \mathbf{s}_{i}+\mathbf{n}_{i}$ and $\mathbf{x}_{i}=F_{i} \mathbf{s}_{i}$, and the standard Kalman filter (Maybeck, 1979) is directly applicable.

The performances of the Kalman filter in the linear case have been completely characterized as, for example, in (Maybeck, 1979). If we assume that the $\mathbf{n}_{\boldsymbol{i}}$ and the $\mathbf{x}_{\boldsymbol{i}}$ are Gaussian then, among all possible estimators, the Kalman filter provides the one with minimum variance, i.e., which minimizes

$$
E\left[\left(\mathbf{s}_{i}-\widehat{\mathbf{s}}_{i}\right)^{t}\left(\mathbf{s}_{i}-\widehat{\mathbf{s}}_{i}\right)\right]
$$

If we do not assume Gaussianness, among all possible linear estimators (those which are computed as linear functions of the measurements), the Kalman filter also computes the one with minimum variance. Note that in this case there may exist nonlinear estimators that yield better results, i.e a smaller variance.

In that sense, in the case of linear state and measurement equations, the Gaussian assumption is unnecessary.

If $\mathbf{h}_{\boldsymbol{i}}\left(\mathbf{s}_{\boldsymbol{i}}\right)$ is not linear or if a linear relationship between $\mathbf{x}_{\boldsymbol{i}}$ and $\mathbf{s}_{\boldsymbol{i}}$ does not exist, the so-called extended Kalman filter (EKF) can be applied. The EKF approach is to apply the standard Kalman filter (for linear systems) to nonlinear systems with additive white noise by continually updating a linearization around the previous state estimate, starting with an initial guess. In other words, we only consider linear Taylor approximations of the state equation at the previous state estimate and of the measurement equation at the corresponding predicted state. This approach gives a 
The relation between $s$ and $x$ described by Equation 29 is not linear. In order to apply the EKF algorithm, it is necessary to compute the derivatives of $f(x, s)$ with respect to $s$ and $x$. It is easy to show that

$$
\begin{aligned}
\frac{\partial \mathbf{f}}{\partial \mathbf{s}} & =\left[\begin{array}{ccc}
\frac{\partial \mathbf{f}_{1}}{\partial \Psi_{1}} & \mathbf{0} & \mathbf{0} \\
\frac{\partial \mathbf{f}_{2}}{\partial \boldsymbol{\omega}} & V & A
\end{array}\right], \\
\frac{\partial \mathbf{f}}{\partial \mathbf{x}} & =\left[\begin{array}{cccc}
\frac{\partial \mathbf{f}_{1}}{\partial \psi_{1}} & \mathbf{0} & -\mathbf{I}_{2} & \mathbf{0} \\
\mathbf{0} & W & \mathbf{0} & -\mathbf{I}_{3}
\end{array}\right],
\end{aligned}
$$

where

$$
\begin{aligned}
\frac{\partial \mathbf{f}_{1}}{\partial \omega} & =\frac{\partial g}{\partial \mathbf{u}_{1}^{\prime}} \frac{\partial\left(W \mathbf{u}_{1}\right)}{\partial \omega} \\
\frac{\partial \mathbf{f}_{2}}{\partial \omega} & =\frac{\partial\left(W \mathbf{m}_{1}\right)}{\partial \omega}+\frac{\partial(V \mathbf{v})}{\partial \omega}+\frac{\partial(A \mathbf{a})}{\partial \omega} \\
\frac{\partial \mathbf{f}_{1}}{\partial \phi_{1}} & =\frac{\partial g}{\partial \mathbf{u}_{1}^{\prime}} W \frac{\partial h}{\partial \psi_{1}}
\end{aligned}
$$

with $\mathbf{u}_{1}=h\left(\boldsymbol{\psi}_{1}\right)$ and $\mathbf{u}_{1}^{\prime}=W \mathbf{u}_{1} \cdot \frac{\partial g}{\partial \mathbf{u}_{1}^{\prime}}$ can be computed from Formula 3. $\frac{\partial h}{\partial \boldsymbol{\psi}_{1}}$ can be computed from Formula 2. Since $\frac{\partial\left(W \mathbf{u}_{1}\right)}{\partial \boldsymbol{\omega}}$ has the same formula as $\frac{\partial\left(W \mathbf{m}_{1}\right)}{\partial \boldsymbol{\omega}}$, we then only need to compute $\frac{\partial\left(W \mathbf{u}_{1}\right)}{\partial \boldsymbol{\omega}}, \frac{\partial(V \mathbf{v})}{\partial \boldsymbol{\omega}}$ and $\frac{\partial(A \mathbf{a})}{\partial \boldsymbol{\omega}}$.

After some simple computations, we get:

$$
\begin{aligned}
\frac{\partial(W \mathbf{u})}{\partial \boldsymbol{\omega}}= & -\frac{\sin (\theta \Delta t)}{\theta} \widetilde{\mathbf{u}}+\frac{\theta \Delta t \cos (\theta \Delta t)-\sin (\theta \Delta t)}{\theta^{3}}(\tilde{\boldsymbol{\omega}} \mathbf{u}) \boldsymbol{\omega}^{t} \\
& +\frac{\theta \Delta t \sin (\theta \Delta t)-2(1-\cos (\theta \Delta t))}{\theta^{4}}(\tilde{\boldsymbol{\omega}}(\tilde{\boldsymbol{\omega}} \mathbf{u})) \boldsymbol{\omega}^{t} \\
& +\frac{1-\cos (\theta \Delta t)}{\theta^{2}}\left[-\widetilde{\tilde{\omega} \mathbf{u}}+(\boldsymbol{\omega} \cdot \mathbf{u}) \mathbf{I}_{3}-\mathbf{u} \boldsymbol{\omega}^{t}\right] \\
\frac{\partial(V \mathbf{v})}{\partial \boldsymbol{\omega}}= & -\frac{1-\cos (\theta \Delta t)}{\theta^{2}} \tilde{\mathbf{v}}+\frac{\theta \Delta t \sin (\theta \Delta t)-2(1-\cos (\theta \Delta t))}{\theta^{4}}(\tilde{\boldsymbol{\omega}} \mathbf{v}) \boldsymbol{\omega}^{t} \\
& +\frac{3 \sin (\theta \Delta t)-\theta \Delta t(2+\cos (\theta \Delta t))}{\theta^{5}}(\tilde{\boldsymbol{\omega}}(\tilde{\boldsymbol{\omega}} \mathbf{v})) \boldsymbol{\omega}^{t} \\
& +\frac{\theta \Delta t-\sin (\theta \Delta t)}{\theta^{3}}\left[-\widetilde{\tilde{\omega} \mathbf{v}}+(\boldsymbol{\omega} \cdot \mathbf{v}) \mathbf{I}_{3}-\mathbf{v} \boldsymbol{\omega}^{t}\right], \\
\frac{\partial(A \mathbf{a})}{\partial \boldsymbol{\omega}}= & -\frac{\theta \Delta t-\sin (\theta \Delta t)}{\theta^{3}} \tilde{\mathbf{a}}+\frac{3 \sin (\theta \Delta t)-\theta \Delta t(2+\cos (\theta \Delta t))}{\theta^{5}}(\tilde{\boldsymbol{\omega}} \mathbf{a}) \boldsymbol{\omega}^{t} \\
& +\frac{4(1-\cos (\theta \Delta t))-(\theta \Delta t)^{2}-\theta \Delta t \sin (\theta \Delta t)}{\theta^{6}}(\tilde{\boldsymbol{\omega}}(\tilde{\boldsymbol{\omega}} \mathbf{a})) \boldsymbol{\omega}^{t} \\
& +\frac{(\theta \Delta t)^{2}-2(1-\cos (\theta \Delta t))}{2 \theta^{4}}\left[-\widetilde{\tilde{\omega} \mathbf{a}}+(\boldsymbol{\omega} \cdot \mathbf{a}) \mathbf{I}_{3}-\mathbf{a} \boldsymbol{\omega}^{t}\right] .
\end{aligned}
$$

where $\boldsymbol{\omega} \cdot \mathbf{u}$ denotes the inner product of the two vectors $\boldsymbol{\omega}$ and $\mathbf{u}$.

When a token matches a segment in the current frame, we use the above formalism to update its kinematic parameters. The same process is applied to each token. 


\section{Matching Segments}

In this section, we describe how to match a token being tracked to a segment in the current frame. The matching technique is based on the Mahalanobis distance, which can be considered as an Euclidean distance in parameter space weighted by uncertainty.

\subsection{Prediction of a Token}

Let $\mathbf{z}=\left[\boldsymbol{\psi}^{t}, \mathbf{m}^{t}\right]^{t}$ be the parameter vector of the token being tracked. The token kinematic parameters are $\left[\boldsymbol{\omega}^{t}, \mathbf{v}^{t}, \mathbf{a}^{t}\right]^{t}$. We can use them to predict its parameter vector $\widehat{\mathbf{z}}=\left[\widehat{\boldsymbol{\psi}}^{t}, \widehat{\mathbf{m}}^{t}\right]^{t}$ at the next time instant:

$$
\left\{\begin{aligned}
\widehat{\psi} & =g(W h(\boldsymbol{\psi})), \\
\widehat{\mathbf{m}} & =W \mathbf{m}+V \mathbf{v}+A \mathbf{a},
\end{aligned}\right.
$$

where the functions $g$ and $h$ are defined as in Equation 28 in Section 7. Due to noise from multiple sources, it is very unlikely that a segment can be found with exactly the parameters $\widehat{\mathbf{z}}=\left[\widehat{\boldsymbol{\psi}}^{t}, \widehat{\mathbf{m}}^{t}\right]^{t}$ and we have to design a matching strategy.

\subsection{Matching Criterion}

Let $\left\{\cdots,\left[\boldsymbol{\psi}_{i}^{t}, \mathbf{m}_{i}^{t}\right], \cdots\right\}$ be the set of observed segments in the scene and $\left[\widehat{\boldsymbol{\psi}}^{t}, \widehat{\mathbf{m}}^{t}\right]^{t}$ be the expected segment. All segments have their measures of uncertainty attached (covariance matrices): $\left\{\cdots, \Lambda_{i}, \cdots\right\}$ and $\Lambda_{\text {token }} . \Lambda_{\text {token }}$ is the covariance matrix of the predicted parameters $\widehat{\mathbf{z}}=\left[\widehat{\boldsymbol{\psi}}^{t}, \widehat{\mathbf{m}}^{t}\right]^{t}$ of the token being tracked, whose computation is given below. The Mahalanobis distance between the expected segment to each segment in the current frame is then given by

$$
d_{i}^{\mathrm{M}}=\mathbf{r}_{i}^{t} \Lambda_{\mathbf{r}_{i}}^{-1} \mathbf{r}_{i}
$$

where

$$
\begin{aligned}
\mathbf{r}_{i} & =\left[\begin{array}{c}
\psi_{i}-\hat{\psi} \\
\mathbf{m}_{i}-\widehat{\mathbf{m}}
\end{array}\right], \\
\Lambda_{\mathbf{r}_{i}} & =\Lambda_{i}+\Lambda_{\text {token }} .
\end{aligned}
$$

The variable $d_{i}^{\mathrm{M}}$ is a scalar random variable following a $\chi^{2}$ distribution with 5 degrees of freedom.

By looking up the $\chi^{2}$ distribution table, we can choose an appropriate threshold $\epsilon$ on the Mahalanobis distance. For example, we can take $\epsilon=11.07$ which corresponds to a probability of $95 \%$ to have the distance $d_{i}^{\mathrm{M}}$ less than $\epsilon$ if the match is correct. Thus segments in the current frame can be considered as plausible matches, if they verify the inequality:

$$
d_{i}^{\mathrm{M}}<\epsilon
$$

Using the above technique, a token may have multiple matches in the current frame. This problem has been described in Section 3.

Before we compute the Mahalanobis distance (Equation 36), we must take care of the discontinuity of $\phi$ when a segment is nearly parallel to the plane $y=0$ (see section 4). The idea is the following. If a segment is represented by $\psi=[\phi, \theta]^{t}$, it is also represented by $[\phi-2 \pi, \theta]^{t}$. Therefore, when comparing the representations of two segments $S$ and $S^{\prime}$, we perform the following tests and actions. If $\phi<\pi / 2$ and $\phi^{\prime}>3 \pi / 2$, then set $\phi^{\prime}$ to be $\phi^{\prime}-2 \pi$; else if $\phi>3 \pi / 2$ and $\phi^{\prime}<\pi / 2$, then 
simple and efficient algorithm to handle a nonlinear model. However, convergence to a reasonable estimate may not be achieved if the initial guess is poor or if the dist:arbances are so large that the linearization is inadequate to describe the system. Contrarily to the linear case, there are no optimality results for the EKF, but note that the Gaussian assumption is also unnecessary.

The measurement equation $\mathbf{f}_{i}\left(\mathbf{x}_{i}, \mathbf{s}_{\mathbf{i}}\right)=0$ is first linearized to obtain a new measurement equar tion

$$
\mathbf{y}_{i}=M_{i} \mathbf{s}_{i}+\mathbf{v}_{i},
$$

where $y_{i}$ is the new measurement vector, $v_{i}$ is the noise vector of the new measurement, and $M_{i}$ is the linearized transformation matrix. They are given by

$$
\begin{aligned}
M_{i} & =\frac{\partial \mathbf{f}_{i}\left(\mathbf{x}_{i}, \mathbf{s}_{i}\right)}{\partial \mathbf{s}_{i}}, \\
\mathbf{y}_{i} & =-\mathbf{f}_{i}\left(\mathbf{x}_{i}, \mathbf{s}_{i}\right)+\frac{\partial \mathbf{f}_{i}\left(\mathbf{x}_{i}, \mathbf{s}_{i}\right)}{\partial \mathbf{s}_{i}} \mathbf{s}_{i}, \\
E\left[\mathbf{v}_{i}\right] & =0 \\
E\left[\mathbf{v}_{i} \mathbf{v}_{i}^{t}\right] & =\frac{\partial \mathbf{f}_{i}\left(\mathbf{x}_{i}, \mathbf{s}_{i}\right)}{\partial \mathbf{x}_{i}} \Lambda_{\mathbf{x}_{i}} \frac{\partial \mathbf{f}_{i}\left(\mathbf{x}_{i}, \mathbf{s}_{i}\right)^{t}}{\partial \mathbf{x}_{i}} \triangleq V_{i} .
\end{aligned}
$$

The partial derivatives are evaluated at $\mathbf{s}_{i}=\widehat{\mathbf{s}}_{\mathbf{i} \mid i-1}$ and $\mathbf{x}_{i}=\overline{\mathbf{x}}_{\boldsymbol{i}}$. The extended Kalman filter equations are given as follows:

\section{Algorithm: Extended Kalman Filter}

- State prediction:

$$
\hat{\mathbf{s}}_{i \mid i-1}=\mathbf{h}_{\mathbf{i}}\left(\hat{\mathbf{s}}_{i-1}\right)
$$

- Prediction of the state covariance matrix:

$$
P_{i \mid i-1}=\frac{\partial \mathbf{h}_{i}}{\partial \mathbf{s}_{i}} P_{i-1} \frac{\partial \mathbf{h}_{i}{ }^{t}}{\partial \mathbf{s}_{i}}+Q_{i-1}
$$

- Kalman gain matrix:

$$
K_{i}=P_{i \mid i-1} M_{i}^{t}\left(M_{i} P_{i \mid i-1} M_{i}^{t}+V_{i}\right)^{-1}
$$

- Update of the state estimation:

$$
\widehat{\mathbf{s}}_{i}=\widehat{\mathbf{s}}_{i \mid i-1}+K_{i}\left(\mathbf{y}_{i}-M_{i} \widehat{s}_{i \mid i-1}\right)=\widehat{s}_{i \mid i-1}-K_{i} \mathbf{f}_{i}\left(\overline{\mathbf{x}}_{i}, \widehat{\mathbf{s}}_{i \mid i-1}\right)
$$

- Update of the covariance matrix of states:

- Initialization:

$$
P_{i}=\left(\mathrm{I}-K_{i} M_{i}\right) P_{i \mid i-1}
$$

$$
P_{0 \mid 0}=\Lambda_{\mathbf{s}_{0}} \quad \widehat{\mathbf{s}}_{0 \mid 0}=E\left[\mathbf{s}_{0}\right]
$$

\section{Formulation of Motion Tracking Problem for EKF Approach}

In this section, we formulate the motion tracking problem in such a way that we can apply the extended Kalman filter formulation of the preceding section. The token is assumed to undergo a motion with constant angular velocity and constant translational acceleration (see Theorem 2 ). We are given a sequence of stereo frames taken at $t_{0}, t_{1}, \cdots, t_{i-1}, t_{i}, \cdots$, such that the interval between $t_{i-1}$ and $t_{i}$ is constant and is denoted by $\Delta t$. 


\subsection{State Transition Equation}

Let the angular velocity at time $t_{i}$ be $\omega_{i}$, the translational velocity $\mathbf{v}_{i}$ and the translational acceleration $\mathbf{a}_{i}$. Define the state vector as

$$
\mathbf{s}_{i}=\left[\begin{array}{lll}
\omega_{i} & \mathbf{v}_{i} & \mathbf{a}_{i}
\end{array}\right]^{t}
$$

The state transition equation can be written as:

$$
\mathbf{s}_{i}=H \mathbf{s}_{i-1}+\mathbf{n}_{i-1}
$$

where

$$
H=\left[\begin{array}{ccc}
\mathbf{I}_{3} & \mathbf{0} & \mathbf{0} \\
\mathbf{0} & \mathbf{I}_{3} & \mathbf{I}_{3} \Delta t \\
\mathbf{0} & \mathbf{0} & \mathbf{I}_{3}
\end{array}\right]
$$

We then replace $\frac{\partial \mathbf{h}_{i}}{\partial \mathbf{s}_{i}}$ in the EKF Algorithm by $H$, since the transition function is linear. The $\mathbf{n}_{i-1}$ in Equation 26 is the random disturbance, with

$$
\mathrm{E}\left[\mathbf{n}_{i-1}\right]=0 \quad \text { and } \quad \Lambda_{\mathbf{n}_{i-1}}=Q_{i-1}
$$

$\mathbf{n}_{i}$ is used first to model noise due to, for example, vibration of objects during motion. But our constant acceleration kinematic model is also in general only an approximation. By adding $\mathbf{n}_{\mathbf{i}-\mathbf{1}}$ in the dynamic model, we can partially take the approximation error in to account.

\subsection{Measurement Equations}

As described in Section 4, a segment $S$ is represented by $\boldsymbol{\psi}$ and $\mathbf{m}$ and their covariance matrices. Suppose a match $\left\{S_{1}, S_{2}\right\}$ is given, where $S_{1}$ occurs at time $t_{i-1}$ and $S_{2}$ at time $t_{i}$. We define the measurement vector as

$$
\mathbf{x}=\left[\begin{array}{llll}
\psi_{1}^{t} & \mathbf{m}_{1}^{t} & \psi_{2}^{t} & \mathbf{m}_{2}^{t}
\end{array}\right]^{t}
$$

From Theorem 2, we have the following equation:

$$
\mathbf{m}_{2}=W \mathbf{m}_{1}+V \mathbf{v}+A \mathbf{a} .
$$

Let $\mathbf{u}_{1}$ be the unit direction vector of segment $S_{1}$ and $\mathbf{u}_{2}$ that of segment $S_{2}$. We have the following relation:

$$
\mathbf{u}_{2}=W \mathbf{u}_{1} \text {. }
$$

If we define two functions $g$ and $h$ to relate $\psi$ and $\mathbf{u}$ together (see Equations 2 and 3 ) so that

$$
\boldsymbol{\psi}=g(\mathbf{u}) \text { and } \mathbf{u}=h(\boldsymbol{\psi}),
$$

then we have the following measurement equation

$$
\mathbf{f}(\mathbf{x}, \mathbf{s})=\left[\begin{array}{c}
g\left(W h\left(\psi_{1}\right)\right)-\psi_{2} \\
W \mathbf{m}_{1}+V \mathbf{v}+A \mathbf{a}-\mathbf{m}_{2}
\end{array}\right]=\mathbf{0} .
$$

This is a 5-dimensional vector equation. In the following, the first two elements in $\mathbf{f}(\mathbf{x}, \mathbf{s})$ are denoted by $f_{1}$ and the last three elements by $f_{2}$. 
set $\phi$ to be $\phi-2 \pi$; else do nothing. Notice that adding a constant to a random variable does not affect its covariance matrix.

Note that the fact that $\phi$ is discontinuous is only an artifact of the representation used. The right way to avoid this kind of problems is to use the notions of manifold and maps (Faugeras, 1991) which are outside the scope of this paper.

Now we return to the problem of computing $\Lambda_{\text {token }}$. Given the original parameters of the token $\left[\boldsymbol{\psi}^{t}, \mathbf{m}^{t}\right]^{t}$ and their covariance matrix $\Lambda_{(\boldsymbol{\psi}, \mathbf{m})}$, and given the kinematic parameters $\left[\boldsymbol{\omega}^{t}, \mathbf{v}^{t}, \mathbf{a}^{t}\right]^{t}$ and their covariance matrix $\Lambda_{(\omega, v, a)}$. Under the first order approximation, the covariance matrix of the expected segment is given by

$$
\Lambda_{\text {token }}=J_{(\psi, \mathrm{m})} \Lambda_{(\psi, \mathrm{m})} J_{(\psi, \mathrm{m})}^{t}+J_{(\omega, \mathrm{v}, \mathrm{a})} \Lambda_{(\omega, \mathrm{v}, \mathrm{a})} J_{(\omega, \mathrm{v}, \mathrm{a})}^{t}
$$

From Equation 35, the Jacobian matrix with respect to $\left[\psi^{t}, \mathbf{m}^{t}\right]^{t}$ is

$$
J_{(\psi, \mathbf{m})}=\left[\begin{array}{cc}
\frac{\partial g}{\partial \mathbf{u}^{\prime}} W \frac{\partial h}{\partial \psi} & \mathbf{0} \\
\mathbf{0} & W
\end{array}\right]
$$

and the Jacobian matrix with respect to $\left[\boldsymbol{\omega}^{t}, \mathbf{v}^{t}, \mathbf{a}^{t}\right]^{t}$ is

$$
J_{(\boldsymbol{\omega}, \mathbf{v}, \mathbf{a})}=\left[\begin{array}{ccc}
\frac{\partial g}{\partial \mathbf{u}^{\prime}} \frac{\partial(W \mathbf{u})}{\partial \boldsymbol{\omega}} & \mathbf{0} & \mathbf{0} \\
\frac{\partial(W \mathbf{m})}{\partial \omega}+\frac{\partial(V \mathbf{v})}{\partial \omega}+\frac{\partial(A \mathbf{a})}{\partial \omega} & V & A
\end{array}\right],
$$

where $\mathbf{u}=h(\boldsymbol{\psi})$ and $\mathbf{u}^{\prime}=W h(\psi)$. All derivatives in the above equations are computed as in Section 7 .

\subsection{Reducing the Complexity by Bucketing Technique}

Although the complexity of matching one segment is linear in the number of segments present in the current frame, the matching process may be slow, especially when there is a large number of segments. This is because the computation of the Mahalanobis distance is relatively expensive (it involves the inversion of a $5 \times 5$ matrix). If we can compute the distances $d_{i}^{\mathrm{M}}$ of the expected segment to only a subset of segments which are near the expected one, we can considerably speed up the matching process; this can be achieved by the use of bucketing techniques. which are now standard in Computational Geometry (Preparata and Shamos, 1986).

We can apply the bucketing techniques either in 3D space or in 2D space. Bucketing in the image plane of one camera is preferred because it is cheaper. The image plane is partitioned into $m^{2}$ square windows (buckets) $W_{i j}$ (in our implementation, $m=16$ ). To each window $W_{i j}$ we attach the list of segments $\left\{S_{k}\right\}$ intersecting $W_{i j}$. The key idea of bucketing is that on the average the number of segments intersecting a bucket is much smaller, and in practice constant, than the total number of segments in the frame (see for example (Faugeras et al., 1990) for details). Given a predicted token to be matched, we first compute the buckets which the disk defined by the predicted segment intersects. The disk is defined as follows: its center coincides with the midpoint of the predicted segment and its diameter equals its length plus a number corresponding to the projected uncertainty of its midpoint. Again, the idea is that, on the average, this disk will intersect a small number of buckets, except for a token which just appeared. Since we initialize such a token with a big uncertainty in motion, its corresponding disk is quite big and may intersect many buckets. The 


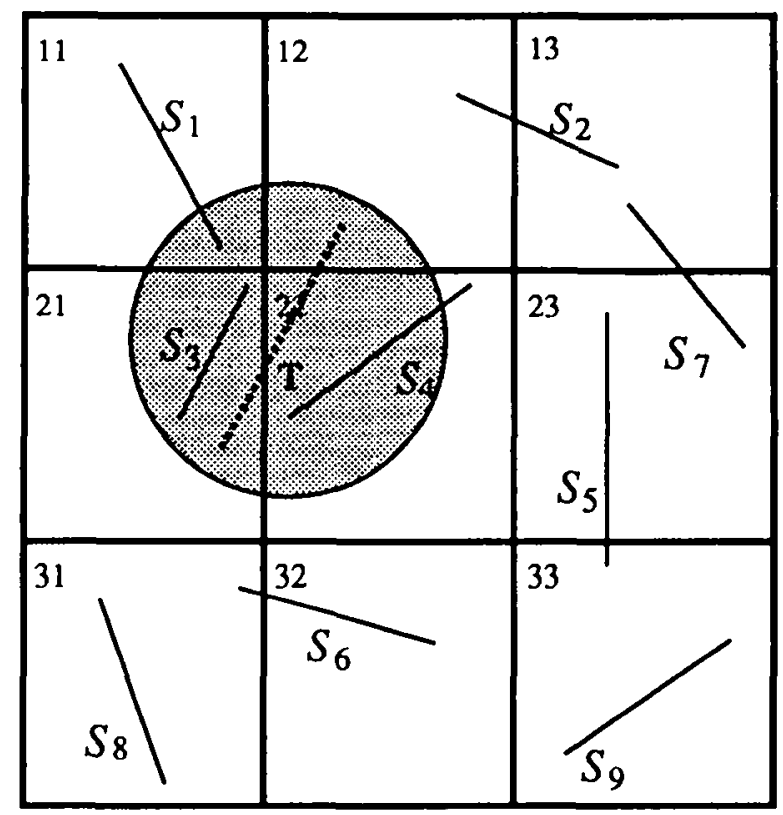

Fig. 6: Bucketing technique

set of potential matching candidates is then the union of the lists of segments intersecting these buckets. This set is now considerably reduced, on the average. Figure 6 illustrates the bucketing technique. In the figure, $T$ is the expected segment. Thanks to the bucketing technique, it finds 4 potential matching candidates $\left\{S_{1}, S_{2}, S_{3}, S_{4}\right\}$ instead of 9 in total. After computing the matching criterion described in the above section, there remain only two candidates $S_{3}$ and $S_{4}$. Note that the computation of buckets can be performed very quickly by an algorithm whose complexity is linear in the number of segments in the current frame.

\section{Support of Existence}

In this section we describe in detail how the beam search strategy sketched in section 2 makes tracking much more robust by allowing multiple matches.

Indeed, in practice, a token being tracked may find several correspondences in the current frame. The most common strategy is to choose the nearest segment as in (Crowley et al., 1988; Deriche and Faugeras, 1990) and to discard the other possibilities.

Our implementation is based on the work of Bar-Shalom and Fortmann (Bar-Shalom and Fortmann, 1988) and is much less sensitive to false matches. The idea is to keep open the possibility of accepting several or no matches for any given token. But, if tokens never disappear we may rapidly reach a computational explosion. To avoid this we compute for each token a number that we call its support of existence which measures the adequateness of the token with the measurements: if the token has not found any correspondences in a long time then it is bound either to be the result of a false match that happened in the past or to have disappeared from the scene.

We use the notations of Section 8 and denote the sequence of measurements corresponding to 
the token being tracked up to time $t_{k}$ as $Z^{k} \triangleq\left\{z\left(t_{1}\right), \cdots, \mathbf{z}\left(t_{k}\right)\right\}$ in which $\mathbf{z}\left(t_{i}\right)=\left[\psi^{t}\left(t_{i}\right), \mathbf{m}^{t}\left(t_{i}\right)\right]^{t}$ is the parameter vector of the segment observed at time $t_{i}$. Denote the event that $Z^{k}$ yields a correct token, i.e., that its components $z\left(t_{i}\right)$ were produced by the same segment moving in space, by $e \triangleq\left\{Z^{k}\right.$ yields a correct token $\}$. The likelihood function of this sequence yielding a correct token is the joint probability density function (or PDF):

$$
L^{k}(e)=p\left(Z^{k} \mid e\right)=p\left[\mathbf{z}\left(t_{1}\right), \cdots, \mathbf{z}\left(t_{k}\right) \mid e\right] .
$$

From the definition of a conditional PDF, $L^{k}(e)$ can be written as

$$
\begin{aligned}
L^{k}(e) & =p\left[Z^{k-1}, \mathbf{z}\left(t_{k}\right) \mid e\right]=p\left[\mathbf{z}\left(t_{k}\right) \mid Z^{k-1}, e\right] p\left[Z^{k-1} \mid e\right] \\
& =\prod_{i=1}^{k} p\left[\mathbf{z}\left(t_{i}\right) \mid Z^{i-1}, e\right]
\end{aligned}
$$

where $Z^{0}$ represents the prior information.

As in Section 8, we denote the measurement residual as $\mathbf{r}$, i.e., $\mathbf{r}_{\mathbf{i}}=\mathbf{z}\left(t_{i}\right)-\widehat{\mathbf{z}}\left(t_{i}\right)$. Then $p\left(\mathbf{r}_{i}\right)=N\left[\mathbf{r}_{i} ; \mathbf{0}, \Lambda_{\mathbf{r}_{i}}\right]$ with $\Lambda_{\mathbf{r}_{i}}=\Lambda_{i}+\Lambda_{\text {token }}$. We use $N[\mathbf{x} ; \overline{\mathbf{x}}, \Lambda]$ to denote the Gaussian density function of the random variable $\mathbf{x}$ with mean $\bar{x}$ and covariance $\Lambda$. We now make the admittedly strong assumption that the $\mathbf{r}_{\boldsymbol{i}}$ are Gaussian and uncorrelated. We thus write:

$$
p\left[\mathbf{z}\left(t_{i}\right) \mid Z^{i-1}, e\right]=N\left[\mathbf{r}_{i} ; \mathbf{0}, \Lambda_{\mathbf{r}_{i}}\right]
$$

It follows that under the previous assumption:

$$
L^{k}(e)=\left[\prod_{i=1}^{k}\left|2 \pi \Lambda_{\mathbf{r}_{i}}\right|^{-1 / 2}\right] \exp \left[-\frac{1}{2} \sum_{i=1}^{k} \mathbf{r}_{i}^{t} \Lambda_{\mathbf{r}_{i}}^{-1} \mathbf{r}_{\mathbf{i}}\right] .
$$

Note that $\mathbf{r}_{i}^{t} \Lambda_{\mathbf{r}_{i}}^{-1} \mathbf{r}_{i}=d_{i}^{M}$ (see Equation 36). The modified $\log$-likelihood function, corresponding to the exponent of $L^{k}(e)$, is defined as

$$
l_{k} \triangleq-2 \ln \left[L^{k}(e) / \prod_{i=1}^{k}\left|2 \pi \Lambda_{\mathbf{r}_{i}}\right|^{-1 / 2}\right]=\sum_{i=1}^{k} d_{i}^{M}
$$

and can be computed recursively as follows:

$$
l_{k}=l_{k-1}+d_{k}^{M}
$$

The last term has a $\chi^{2}$ distribution with $n_{r}=5$ degrees of freedom. Since the $\mathbf{r}_{k}$ are assumed to be independent, $l_{k}$ has a $\chi^{2}$ distribution with $k n_{r}$ degrees of freedom.

The statistical test for deciding that $Z^{k}$ yields a correct token is that the log-likelihood function satisfies

$$
l_{k} \leq \kappa,
$$

where the threshold $\kappa$ is obtained from the $\chi^{2}$ table with $k n_{r}$ degrees of freedom by setting $\operatorname{Pr}\left(\chi_{k n_{r}}^{2} \leq \kappa\right)=\alpha$, where $\alpha$ is typically equal to $95 \%$.

In practice, the test (44) cannot be used for long sequences because the likelihood function is dominated by old measurements and responds very slowly to recent ones. In order to limit the 
"memory" of system, we can multiply the likelihood function at each step by a discount factor $c<1$. This results in the fading-memory likelihood function:

$$
l_{k}=c l_{k-1}+d_{k}^{M}=\sum_{i=1}^{k} c^{k-i} d_{i}^{M} .
$$

The effective memory of $l_{k}$ is now $(1-c)^{-1}$, and in steady state $l_{k}$ is approximately a $\chi^{2}$ random variable with $n_{r}(1+c) /(1-c)$ degrees of freedom, mean $n_{r} /(1-c)$, and variance $2 n_{r} /\left(1-c^{2}\right)$. See (Bar-Shalom and Fortmann, 1988) for the proof. In our implementation, $c=0.75$.

In the above discussion, we assume implicitly that a match is detected at each sampling time. As described earlier, match detection may fail from time to time for a number of reasons. In these cases, it means that:

$$
d_{k}^{M} \geq \epsilon,
$$

as described in Section 8. Thus, if at time $t_{k}$ no match is found, the fit between the prediction and the observation is not very good. But note that even in that case we may still have $l_{k} \leq \kappa$ and the processing of the token will continue. This allows us to cope with problems such as occlusion, disappearance and absence. Of course if the Mahalanobis distances stay over the threshold $\epsilon$ at too many consecutive time instants, i.e., if the token does not find any good match in the scene too often, then $l_{k}$ will go beyond the threshold $\kappa$, and the token will be discarded, as expected. In practice we set $d_{k}^{M}=\alpha \epsilon$ where $\alpha=1.2$ in our implementation.

\section{Grouping Tokens into Objects}

In the previous sections, we have described how to track each token and estimate its kinematic parameters in parallel. In this section, we address the following problem: how can the supervisor group tokens into objects based on their kinematic parameters?

We assume that moving objects in the scene are rigid. From Section 5 , we know that each token belonging to a rigid object must have the same kinematic parameters with respect to a common point. In our algorithm, the kinematic parameters of all tokens are computed with respect to the same point-the origin of the system of reference. We can then define an object as follows: an object is a set of tokens with the same kinematic parameters. Under this definition, two different objects are grouped as a single one if they undergo the same motion.

Of course, the estimated kinematic parameters are uncertain, and have attached to them an uncertainty measure - their covariance matrix. One cannot expect to find two tokens having exactly the same kinematic parameters. The Mahalanobis distance is again used in this case to measure the discrepancy between kinematic parameters. Given two kinematic parameter vectors $s_{1}$ and $s_{2}$ and their covariance matrices $\Lambda_{\mathbf{s}_{1}}$ and $\Lambda_{\mathbf{s}_{2}}$, their Mahalanobis distance is given by

$$
\delta^{M}=\left[\mathbf{s}_{1}-\mathbf{s}_{2}\right]^{t}\left(\Lambda_{\mathbf{B}_{1}}+\Lambda_{\mathbf{B}_{2}}\right)^{-1}\left[\mathbf{s}_{1}-\mathbf{s}_{2}\right]
$$

When $\mathrm{s}=[\boldsymbol{\omega}, \mathbf{v}, \mathbf{a}]^{t}, \delta^{\mathrm{M}}$ is a random scalar following a $\chi^{2}$ distribution with 9 degrees of freedom. By looking up the $\chi^{2}$ distribution table, we can choose an appropriate threshold $\varepsilon$ on the Mahalanobis distance. For example, we can take $\varepsilon=16.92$ which corresponds to a probability of $95 \%$ that the distance $\delta^{\mathrm{M}}$ is less than $\varepsilon$. Thus two tokens can be considered to belong to the same object, if the Mahalanobis distance of their kinematic parameters verifies the inequality:

$$
\delta^{\mathrm{M}}<\varepsilon .
$$


If two tokens are identified to belong to a single object by the above test, we can compute a better estimate $\mathbf{s}$ of the kinematic parameters for the object from those attached to each token by the Kalman filter or simply by the modified Kalman minimum-variance estimator:

$$
\begin{aligned}
\mathbf{s} & =\Lambda_{\mathbf{s}}^{-1}\left(\Lambda_{\mathbf{s}_{1}}^{-1} \mathbf{s}_{1}+\Lambda_{\mathbf{B}_{2}}^{-1} \mathbf{s}_{2}\right) \\
\Lambda_{\mathbf{s}} & =\left(\Lambda_{\mathbf{s}_{1}}^{-1}+\Lambda_{\mathbf{B}_{2}}^{-1}\right)^{-1}
\end{aligned}
$$

where $\Lambda_{\mathbf{B}}$ is the covariance matrix of the new estimate $\mathbf{s}$. We then try to find more tokens compatible with $\mathbf{s}$ and $\Lambda_{\mathbf{s}}$ and update them by Equation 47 . When all tokens have been processed, we get

- an object with a list of tokens associated with it and its kinematic parameters, and

- a list of tokens not yet attached to an object.

The same process is then performed on the list of tokens non-identified to recover new objects, and so forth, until no more object can be identified. We finally obtain all plausible objects in the scene.

\section{Experimental Results}

We have implemented the proposed algorithm on a SUN workstation using the C language. Our program can display and control interactively the motion tracking process. In this section, we provide two experimental results with real data to show the performance of our algorithm. Results with synthetic data has been reported in (Zhang and Faugeras, 1990b). The angular velocity unit is radians/unit-time, and the translational velocity unit is millimeters/unit-time, except when stated otherwise.

In each figure shown below, if there are four pictures, the upper left one is the front view of a 3D frame (i.e., its orthographic projection onto a frontoparallel plane), the upper right one is the top view (i.e., its orthographic projection onto a horizontal plane), and the lower left and right ones form a stereogram to allow the reader to perceive $3 \mathrm{D}$ information by cross-eye fusion. If there are only two, then they are a pair of stereograms.

\subsection{Real Data with Controlled Motion}

The data are acquired as follows: the trinocular stereo rig is in front of a rotating table (at about 2.5 meters). The rotating table has two degrees of freedom: vertical translation and rotation around the vertical. The motion is controlled manually. Boxes are put on the table and will be considered by the program as an object. The table undergoes a general motion, combination of a rotation and a translation parallel to the rotation axis. It rotates of 3 degrees (clockwise, if viewed from the top), and at the same time, translates from bottom to top of 50 millimeters between adjacent frames. Thus, the motion corresponds exactly to the constant velocity model. There are in fact three "objects" in the scene (see Figure 7): the second is the static support of the table, and the third is what is below and attached to the rotating table, and undergoes the same translation but no rotation.

Ten 3D frames have been acquired for this experiment. Each frame contains about 130 3D line segments. Figure 8 displays the images taken by the first camera at $t_{1}$ and $t_{10}$. To show the motion, the first and the second 3D frames are superposed in Figure 9 and the first and the last in Figure 10, together with a pair of stereograms. We find that the data are very noisy even for the 


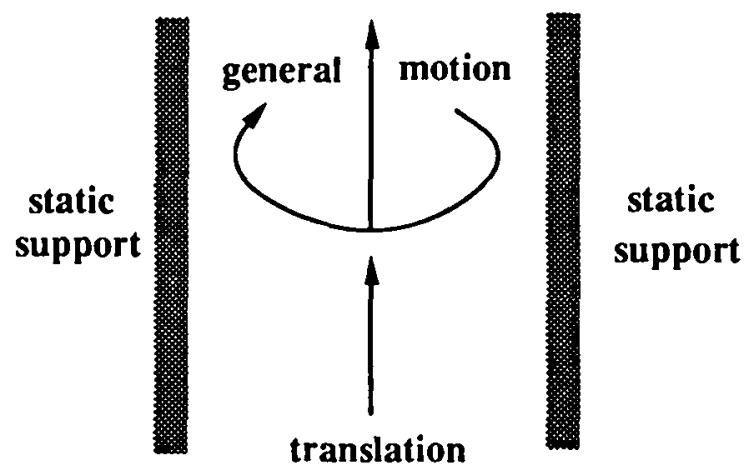

Fig. 7: Description of the rotating table undergoing general motion

static object and that occlusion, appearance, disappearance and absence problems are very severe. We can also observe that the object in translation is not detected until the third frame is taken.

Each segment in the first frame is initialized as a token to be tracked. Since the motion tracking algorithm is recursive, some a priori information on the kinematics is required. A reasonable assumption may be that objects do not move, as the inter-frame motion is expected to be small. The kinematic parameters are thus all initialized to zero, but with fairly large uncertainty: the standard deviation for each angular velocity component is 0.0873 radians/unit-time, and that for each translational velocity component is 150 millimeters/unit-time. The variances on the acceleration components are set to zero (no acceleration).

Those tokens are then predicted for the next instant $t_{2}$ and the predicted tokens are compared with those in the new frame. Of course, since we have assumed no motion, the predicted position and orientation of each token remains unchanged, but its uncertainty changes and becomes very large. Thus Figure 9 displays also the difference between the predicted tokens (in solid lines) and the observed line segments (in dashed lines) at time $t_{2}$. As expected, multiple matches occur for most of tokens. Techniques based only on the best match usually fail at this stage, since the nearest segment is not always the correct match. We retain the two best matches if a token has multiple matches. The token updates its kinematic parameters using its best match. The supervisor initializes a new token by combining the token and its second best match which is used to estimate its kinematic parameters.

We continue the tracking in the same manner. Figure 11 shows the superposition of the predicted segments for $t_{3}$ and the observed segments. As can be observed, more active tokens (in solid lines) exist at this moment: some have been activated due to multiple matches at time $t_{2}$ and some just entered the field of view. We observe that segments belonging to the object undergoing general motion coincide well, and so do the segments of the static support. The segments belonging to the translating object do not coincide well, because they just appeared in the field of view and no information about their kinematics is available. Figure 12 shows the superposition between the predicted segments for $t_{10}$ and the observed segments. Almost all segments are well superposed except those in the middle part. Those segments correspond to the outline of the rotating table which is an ellipse. Using line segments to approximate an ellipse is of course difficult, and this is clearly one of the limitations of our system. One can notice that most of the tokens due to false matching in the preceding instants have disappeared, because they have lost their supports of 

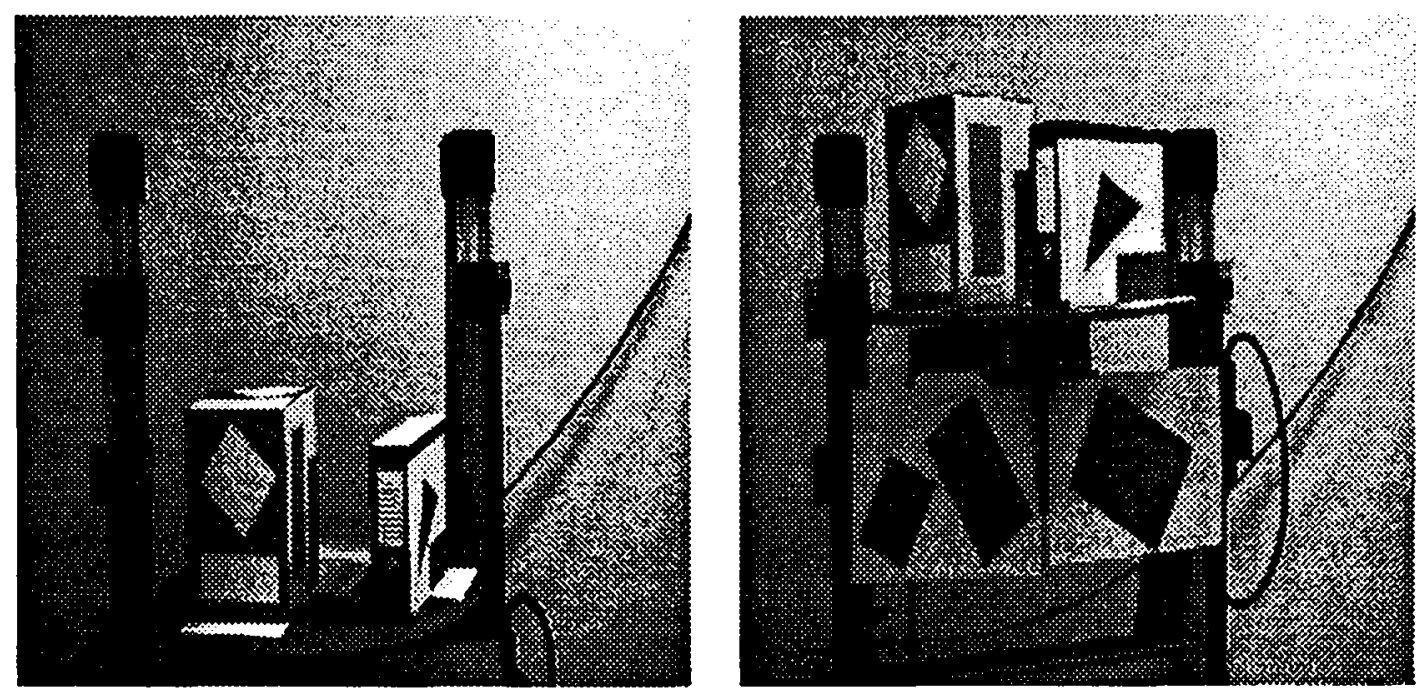

Fig. 8: The images taken by the first camera at $t_{1}$ and $t_{10}$

existence.

The supervisor successfully segments the scene in to 3 groups of objects based on the information of the kinematics of each token. In the following, we show the result of the estimated kinematic parameters of the object undergoing general motion. If we describe the motion in the stereo coordinate system, the kinematic parameter vector is $s=[0.0,5.236 \mathrm{e}-02,0.0, ?,-50, ?]^{t}$. The $x$ and $z$ components of the translational velocity are not known, since because of the calibration method we have used, the rotation axis (i.e., the axis of the cylinder) is not known in the stereo coordinate system. We know the $y$ component of the translational velocity because the rotation. axis is parallel to the $y$ axis. Figure 13 shows the variation of the absolute errors in the estimation of the angular velocity. Figure 14 shows only the error in the estimation of the $y$ component of the translational velocity. The results are good: after a few (four or five) frames, the error for the angular velocity is less than 2.5 milliradians/unit-time (compared with 52.36), and the error for the translational velocity is less than 1.5 millimeters/unit-time (compared with 50 ). The final estimation for this object is

$$
\widehat{\mathrm{s}}=[4.291 \mathrm{e}-04,5.328 \mathrm{e}-02,-7.059 \mathrm{e}-04,-7.387 \mathrm{e}+00,-4.951 \mathrm{e}+01,3.677 \mathrm{e}+01]^{t} .
$$

The relative error of the angular velocity is $2.36 \%$. The angle between the true and estimated axes of rotation is 0.9 degrees. Similar results are observed for the other objects. The final motion estimate corresponding to the translating object is

$$
[3.437 \mathrm{e}-04,1.353 \mathrm{e}-03,-7.303 \mathrm{e}-04,-2.009 \mathrm{e}-01,-4.937 \mathrm{e}+01,8.361 \mathrm{e}-01]^{t} \text {, }
$$

which should be compared with $[0,0,0,0,-50,0]$. The final motion estimate of the static object is

$$
[-2.321 \mathrm{e}-04,5.009 \mathrm{e}-04,2.981 \mathrm{e}-04,2.681 \mathrm{e}-02,-3.399 \mathrm{e}-01,4.799 \mathrm{e}-01]^{t} \text {. }
$$

We now show that the position of the axis of the rotating table in the stereo coordinate system can be computed from the estimated kinematics $\widehat{\mathbf{s}}$ and the known nature of the motion. For a point not on that axis, its trajectory is a helix. For a point on that axis, its trajectory is a straight line. Let a point on the axis be $\mathbf{p}$. From Equation 11, the velocity of $\mathbf{p}$ is given by

$$
\mathbf{v}_{\mathbf{p}}=\mathbf{v}+\boldsymbol{\omega} \times \mathbf{p} .
$$



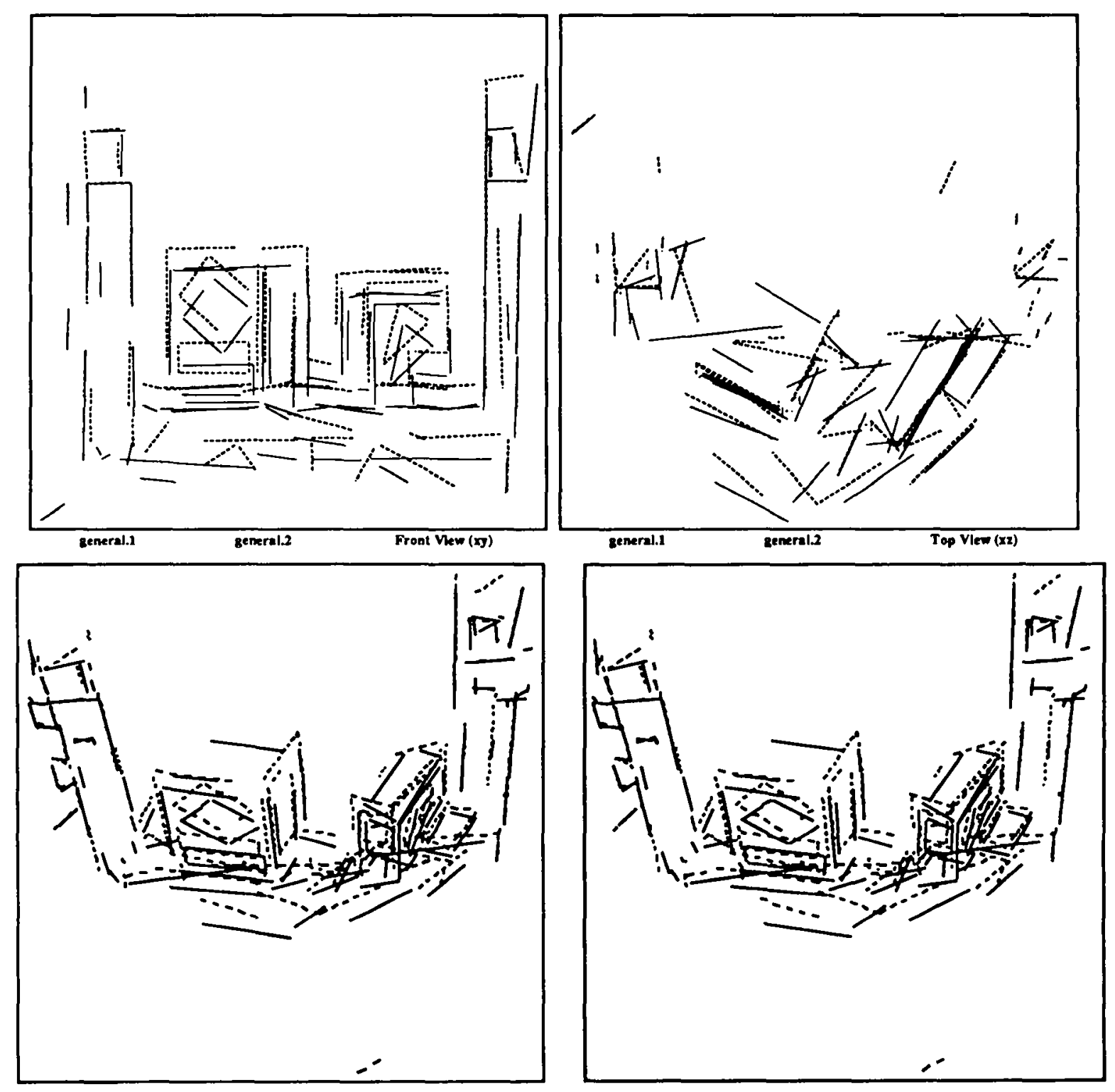

Fig. 9: The superposition of the first (in solid lines) and the second (in dashed lines) frames 

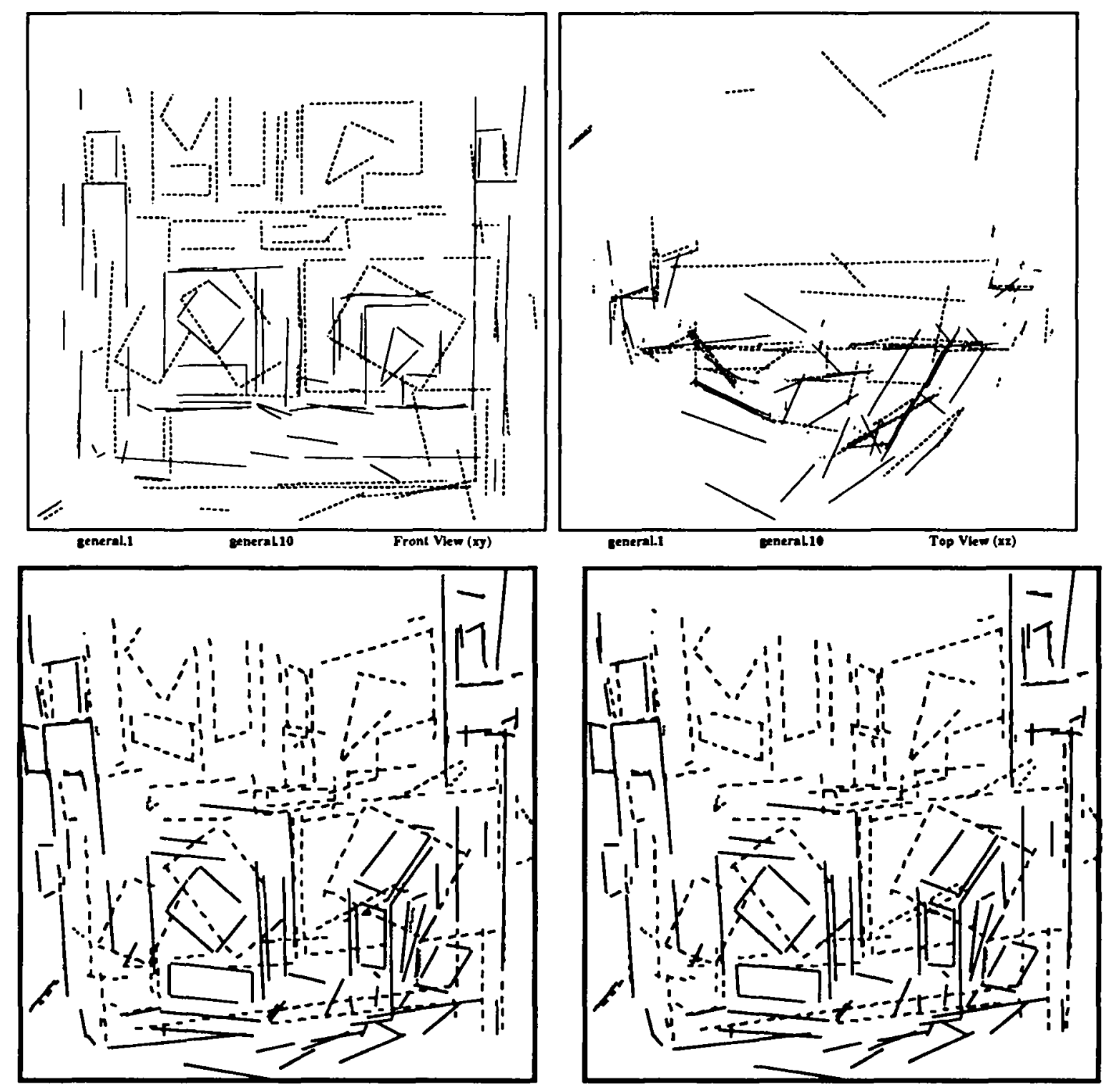

Fig. 10: The superposition of the first (in solid lines) and the last (in dashed lines) frames 
Z. Zhang and O.D. Faugeras. Determining motion from 3D line segments: a comparative study. Image and Vision Computing, 9(1):10-19, February 1991.

Z. Zhang and O.D. Faugeras. Estimation of displacements from two 3D frames obtained from stereo. IEEE Trans. Pattern Analysis and Machine Intelligence, September 1991. accepted, to appear.

Z. Zhang, O.D. Faugeras, and N. Ayache. Analysis of a sequence of stereo scenes containing multiple moving objects using rigidity constraints. In Proc. Second International Conference on Computer Vision, pages 177-186, IEEE, Tampa, Florida, December 1988.

X. Zhuang and R.M. Haralick. Two view motion analysis. In Proc. IEEE Conf. Computer Vision and Pattern Recognition, pages 686-690, San Francisco, California, June 1985. 

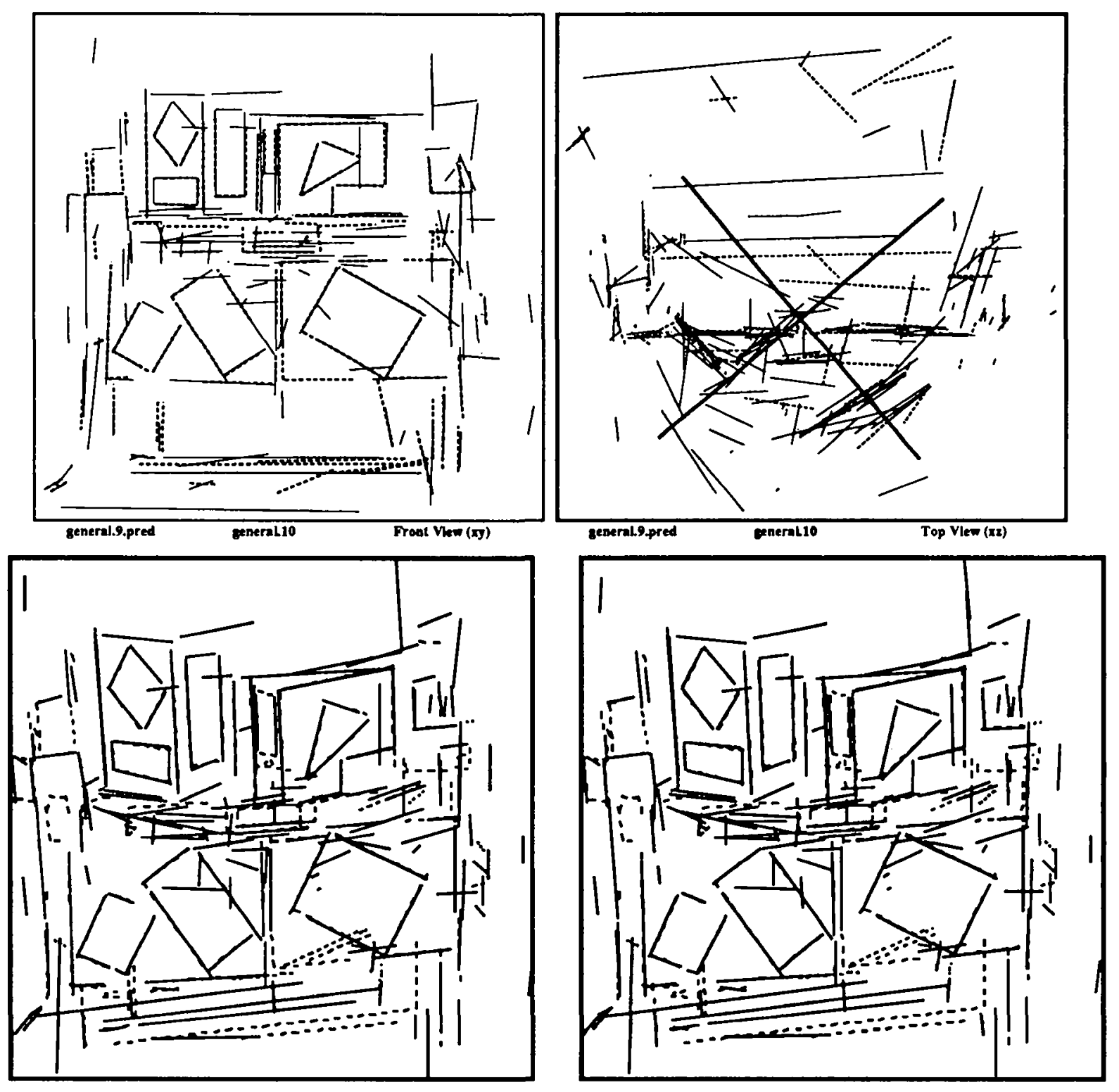

Fig. 12: The superposition of the predicted (in solid lines) and the observed (in dashed lines) segments at time $t_{10}$ 


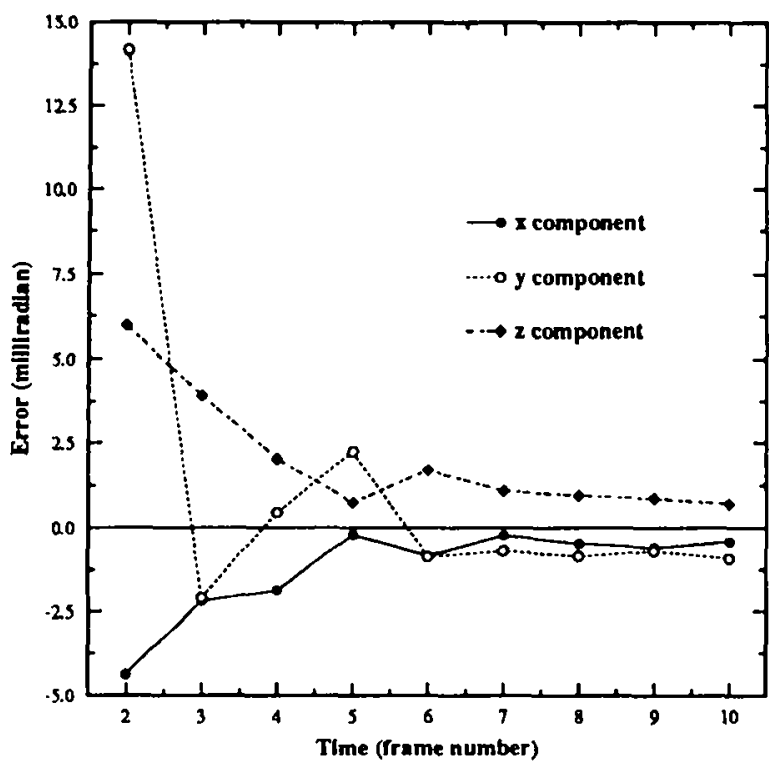

Error evolution in the angular ve-

Fig. 13: locity of the rotating table undergoing general motion

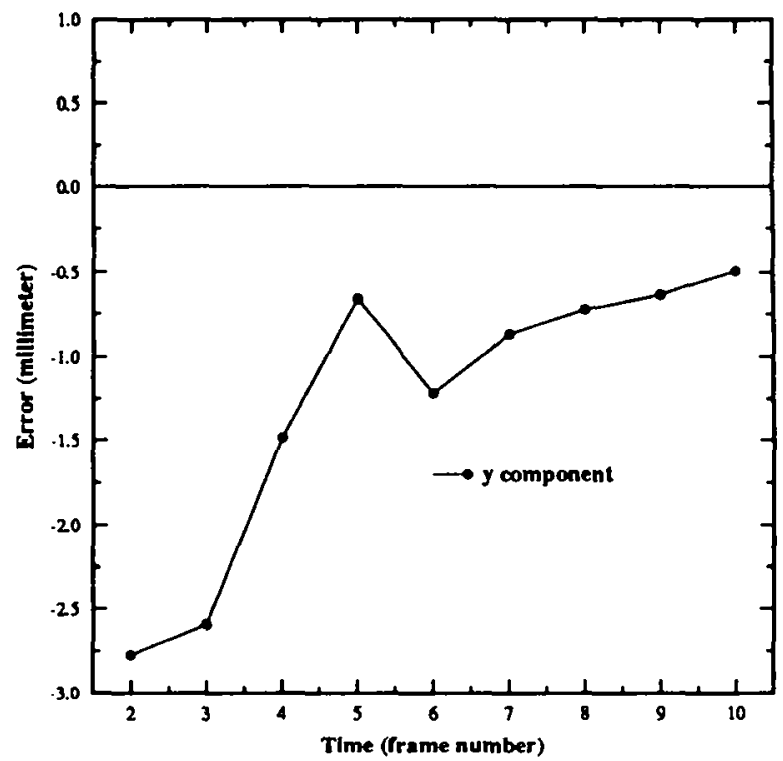

Error evolution in the translational

Fig. 14: velocity of the rotating table undergoing general motion

Since the rotation is about the axis of the table, the velocity $\mathbf{v}_{\mathbf{p}}$ of $\mathbf{p}$ must be parallel to the rotation axis $\boldsymbol{\omega}$, i.e., $\boldsymbol{\omega} \times \mathbf{v}_{\mathrm{p}}=0$. We thus have

$$
\boldsymbol{\omega} \times \mathbf{v}+\boldsymbol{\omega} \times(\boldsymbol{\omega} \times \mathbf{p})=0
$$

Equation 48 gives only two independent scalar equations, because the three components of a crossproduct are not linearly independent. It defines the axis of the table. In order to compute a point on it, we can choose the $y$ component $p_{y}$ of $\mathbf{p}$ equal to 0 , and compute its $x$ and $z$ components: $p_{x}$ and $p_{z}$. In this example, we get $p_{x}=677.68$ and $p_{z}=131.08$. That point is shown at the intersection of the two bold line segments in the top view of Figure 12, and seems roughly correct.

\subsection{Real Data with Uncontrolled Motion}

The data described in this section are acquired as follows. The trinocular stereo rig is mounted on a mobile vehicle, which moves in the laboratory. Objects in the scene are about 2 to 7 meters away from the mobile vehicle. We have taken 15 stereo views while the vehicle was supposed to undergo a translation. Figure 15 displays the images taken by the first camera at $t_{1}$ and $t_{15}$. We have thus reconstructed $153 \mathrm{D}$ frames using our stereo system, each containing around 85 line segments.

Ideally, the motion of the vehicle is a pure translation. If described in our stereo coordinate system, the translation between successive views is about $[-25,0,-98]^{t}$, i.e., a displacement of 100 millimeters. However, due to precision of the mechanical system and slipping of the vehicle wheels, it is naive to believe that the vehicle motion is going to be exactly the requested one. To check this, we have applied an algorithm developed earlier for computing motion between two 3D frames ((Zhang et al., 1988; Zhang and Faugeras, 1991b)) to every successive 3D views. We have found that there is a random rotation of about one degree between successive views and a difference in translation of up to 30 millimeters in $x$ or $z$ direction. To give an idea, we show in Table 1 the $x$ and $z$ components of the computed motions (the sign is omitted). We can remark that the motion 

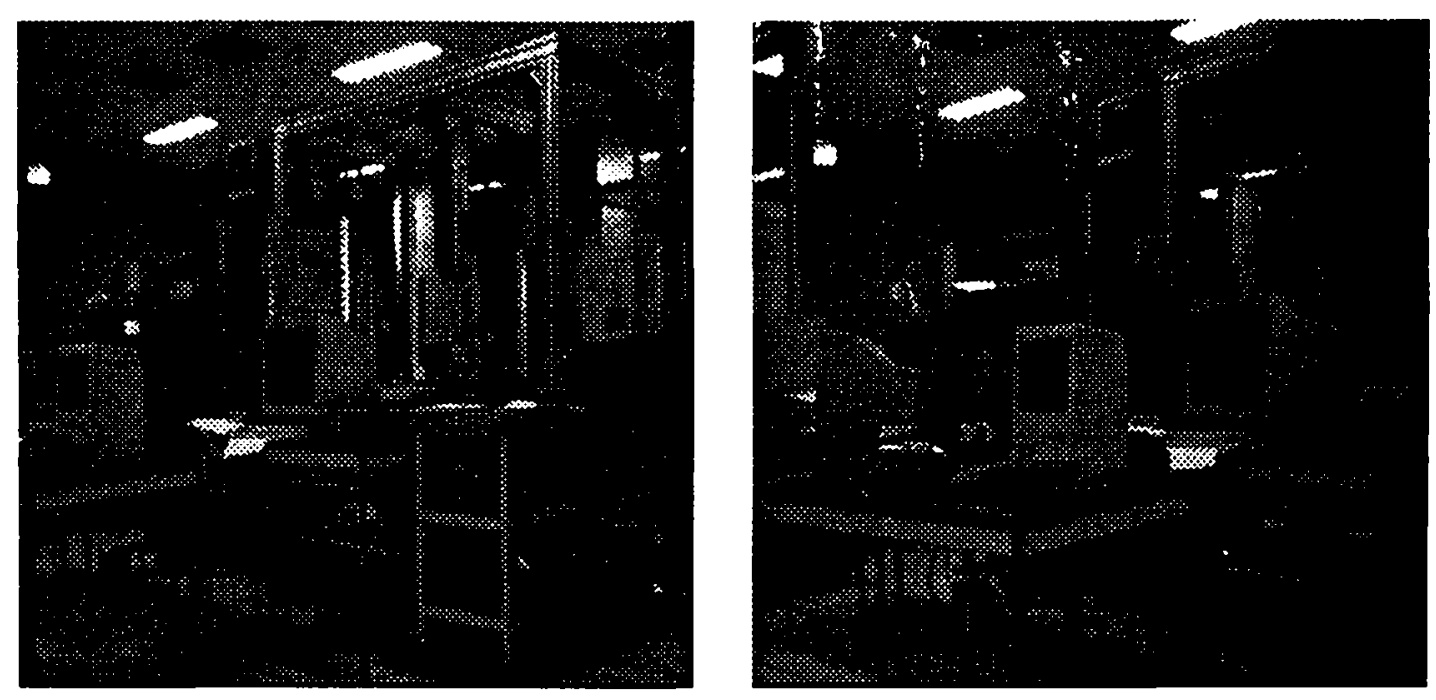

Fig. 15: The images taken by the first camera at $t_{1}$ and $t_{15}$

realized by the mobile vehicle is not very smooth and does not satisfy either the constant velocity or the constant acceleration model. This is our ground truth data for checking the performances of the methods presented in this article.

Table 1: $x$ and $z$ components of the translations between successive views

\begin{tabular}{|c|r|r|r|r|r|r|r|r|r|r|r|r|r|r|}
\hline views & $1-2$ & $2-3$ & $3-4$ & $4-5$ & $5-6$ & $6-7$ & $7-8$ & $8-9$ & $9-10$ & $10-11$ & $11-12$ & $12-13$ & $13-14$ & $14-15$ \\
\hline \hline$x(\mathrm{~mm})$ & 30.8 & 34.8 & 18.7 & 24.7 & 21.0 & 29.5 & 33.1 & 26.2 & 7.1 & 30.7 & 9.8 & 19.9 & 21.1 & 25.4 \\
\hline$z(\mathrm{~mm})$ & 104.1 & 85.7 & 91.1 & 99.7 & 107.7 & 108.7 & 89.4 & 95.0 & 126.8 & 97.3 & 104.8 & 104.3 & 103.7 & 93.8 \\
\hline
\end{tabular}

We apply the techniques described in this article to the above data. As in the previous experiment, each segment in the first frame is initialized as a token to be tracked. The kinematic parameters are all initialized to zero, but with fairly large uncertainties: the standard deviation is 0.0873 radians/unit-time for each angular velocity component, and 150 millimeters/unit-time for each translational velocity component. The variances on the acceleration components are set to zero (no acceleration). The only difference is that the noise term $\mathbf{n}_{\mathbf{i - 1}}$ in the state equation (Equation 26) is not zero. We have added at each step a noise with standard deviation 0.5 degrees/unit-time to the $\boldsymbol{\omega}$ components and a noise with standard deviation 2 millimeters/unit-time to the $v$ components.

The position and orientation of those tokens are then predicted for the next instant $t_{2}$ and the predicted tokens are compared with those in the new frame. Of course, since no motion is assumed, the predicted position and orientation of each token remains unchanged, but its uncertainty changes and becomes very large. Figure 16 displays a stereogram showing the difference between the predicted tokens (in solid lines) and the observed line segments (in dashed lines) at $t_{2}$. The reader can perceive the $3 \mathrm{D}$ information by cross-eye fusion. As expected, multiple matches occur for most of tokens, which are treated in the same way as in the previous experiment.

Figure 17 displays a stereogram showing the superposition of the predicted (in solid lines) and observed (in dashed lines) segments at $t_{3}$. As can be observed from the figure, more active tokens (in solid lines) exist at this moment: some have been activated due to multiple matches 

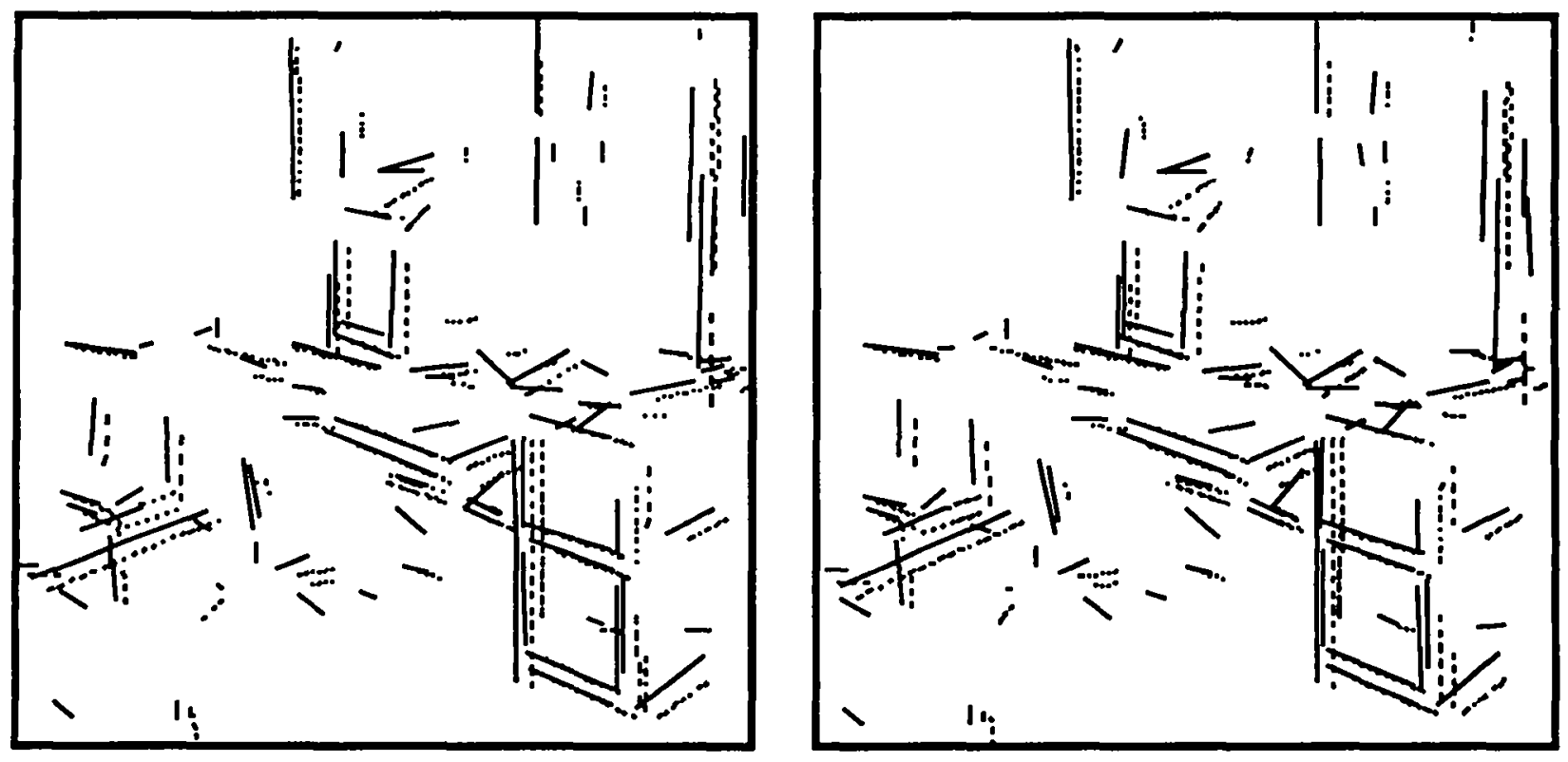

Fig. 16: A stereogram showing the superposition of the predicted (in solid lines) and observed (in dashed lines) segments at $t_{2}$

at time $t_{2}$ and some just entered into the field of view. We observe that most of the tokens have already a good kinematics information since their predictions coincide well with their observations. Unfortunately, this is not always the case. For example, the motion between the ninth and tenth views is not coherent with the global motion (see Table 1). Figure 18 displays a stereogram showing the superposition of the predicted (in solid lines) and observed (in dashed lines) segments at $t_{10}$. We can observe a big difference between the prediction and the observation. After several views, such occasional incoherent motion will be compensated for by the algorithm. Figure 19 displays a stereogram showing the superposition of the predicted (in solid lines) and observed (in dashed lines) segments at $t_{15}$. Quite a good fitting between the prediction and observation can be observed. The final estimate of the angular velocity (in radians/unit-time) is $\left[-1.1 \times 10^{-5}, 2.6 \times 10^{-3},-3.2 \times 10^{-4}\right]^{t}$. The final estimate of the translational velocity (in millimeters/unit-time) is $[-24.5,-1.25,-97.1]^{t}$.

The program runs on a SUN 4/60 workstation. The number of active tokens varies between 110 and 155 , except in the first view ( 89 tokens). The average user time for predicting a token is about 6.7 milliseconds and that for matching and updating a token is about 44.6 milliseconds. That is, if we implement the algorithm on a parallel machine with the same performance as SUN $4 / 60$, the time required to process two frames is a little more than 50 milliseconds. The average user time required to group tokens in to objects is about 2.5 seconds. In the last view, 90 segments have been identified as belonging to the static environment.

\section{Discussion}

In the above discussion about multiple matches we presented the concept of splitting of a token. In fact, two cases of multiple matches should be distinguished. Figure 20 shows such cases. The token $L$ is represented by a thick line segment. It has two matches $S_{1}$ and $S_{2}$ (represented by thin line segments). In the first case, the two matches $S_{1}$ and $S_{2}$ are not collinear. The splitting technique 

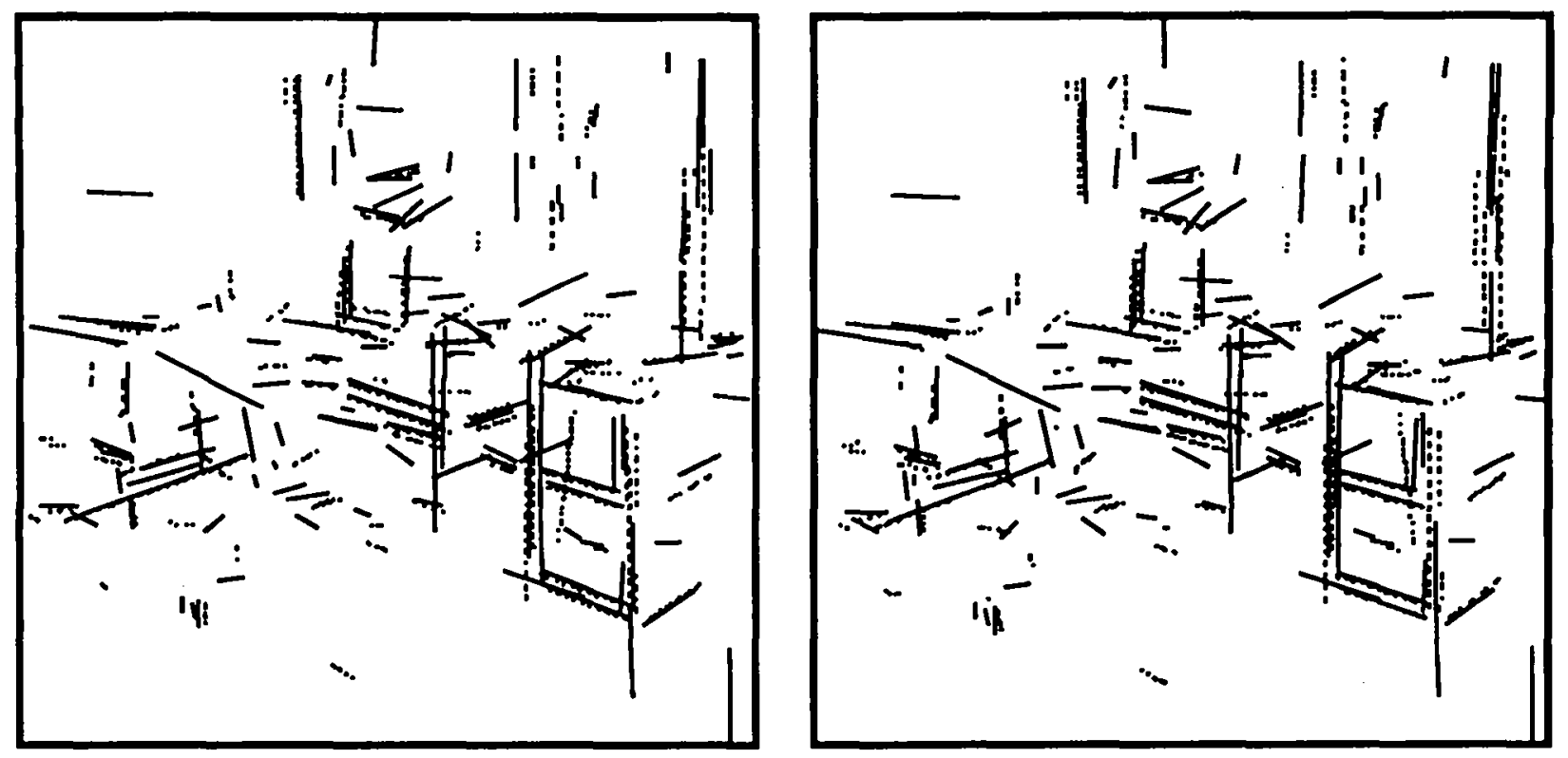

Fig. 17: A stereogram showing the superposition of the predicted (in solid lines) and observed (in dashed lines) segments at $t_{3}$
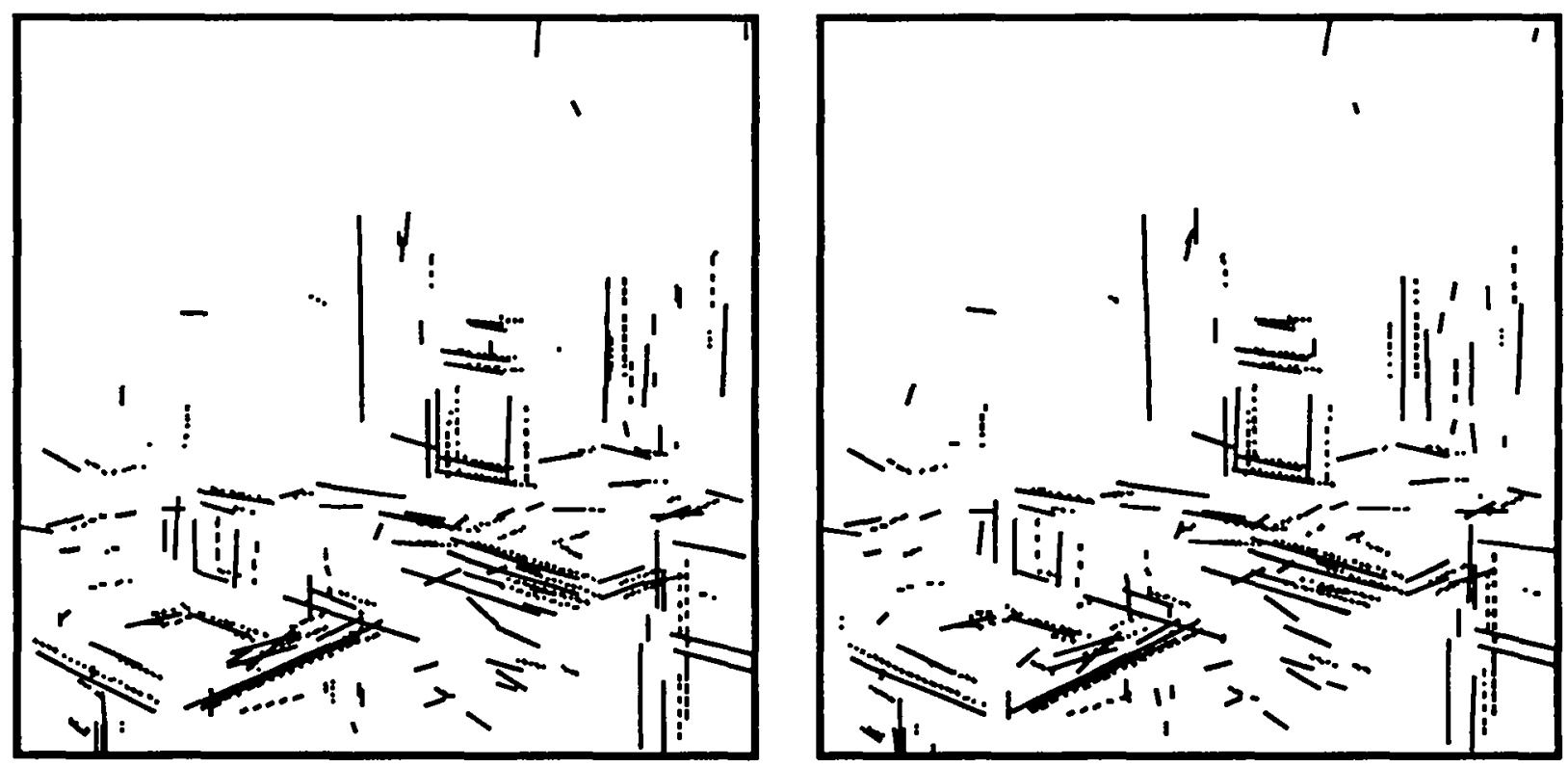

Fig. 18: A stereogram showing the superposition of the predicted (in solid lines) and observed (in dashed lines) segments at $t_{10}$ 

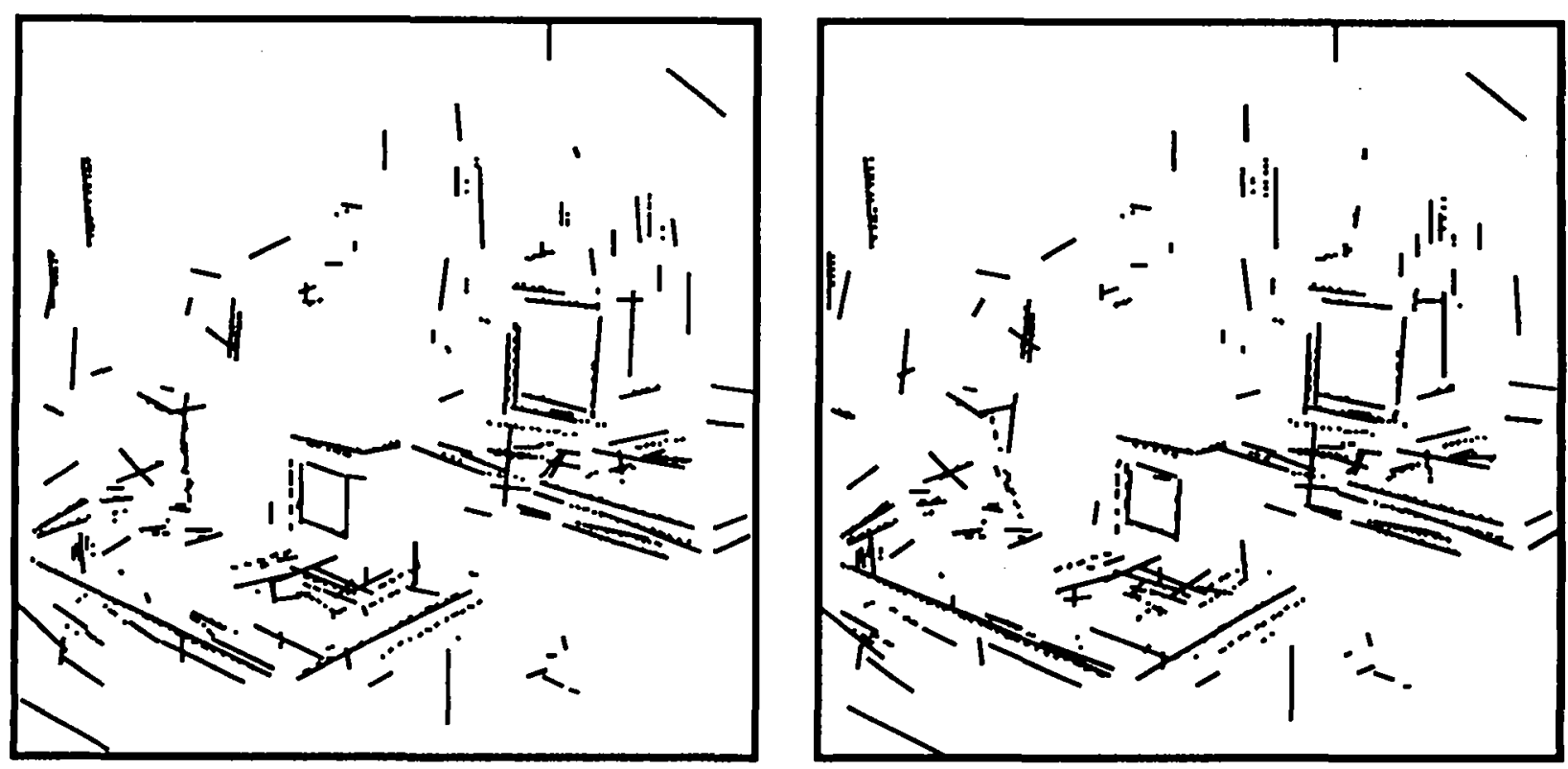

Fig. 19: A stereogram showing the superposition of the predicted (in solid lines) and observed (in dashed lines) segments at $t_{15}$

can be applied to handle the problem. That is, the token $L$ is duplicated, one pursuing the tracking by incorporating $S_{1}$, and another pursuing the tracking by incorporating $S_{2}$.

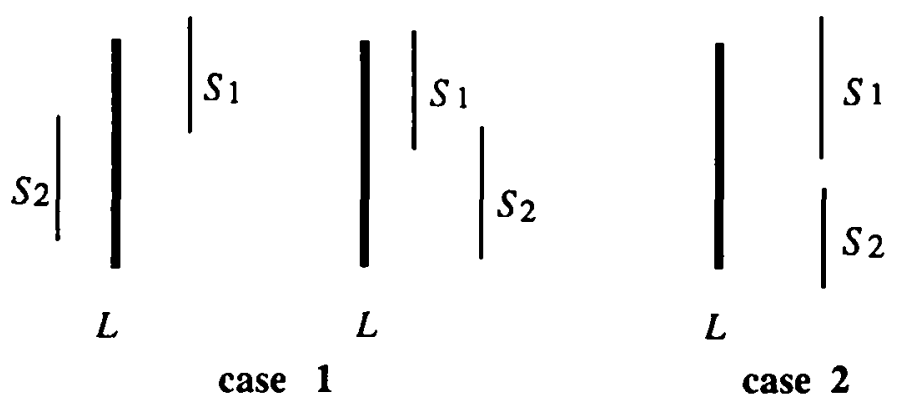

Fig. 20: Two different cases of multiple matches

In the second case, the two matches $S_{1}$ and $S_{2}$ are collinear. Of course, the splitting technique is still applicable, but we do not want to apply it (see below). A reasonable interpretation is that $S_{1}$ and $S_{2}$ are both parts of a single longer segment in space. The segment is broken in two because of the edge detection process, the line segment fitting process or other reasons. Note that not any two collinear segments can be interpreted this way. We use the fact that there exists a token, $L$, which links $S_{1}$ and $S_{2}$ together because it has been matched to both of them. Based on the above consideration, we first merge $S_{1}$ and $S_{2}$ into a single segment $S$ (see (Zhang and Faugeras, 1990a) for the merging technique). The token $L$ continues to track by matching $S$ without splitting.

The merging concept is very important. It can avoid the abnormal growth of the number of 
tokens due to splitting. For example, in an extreme case as shown in Figure 21, we have 2 tokens at $t_{2}, 4$ tokens at $t_{4}, 8$ tokens at $t_{6}, \ldots$.

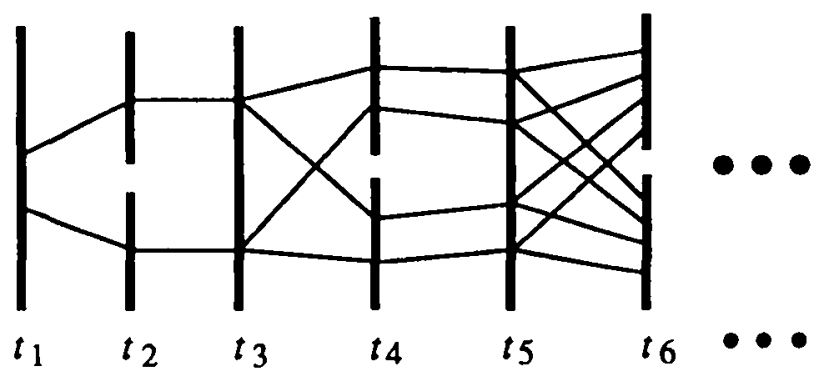

Fig. 21: An example to show the importance of merging

\section{Conclusion}

Our system is based upon three main assumptions. The first one is that the stereo process provides us with features which are fairly stable over time in the sense that they move rigidly in space. Our choice of features has been straight line segments which work well if the environment is close to polyhedral. It is expected that if many curved objects are present the method will break down unless we choose new features but this is an open area for research.

The second assumption is the approximation of the angular velocity by a constant vector and the linear velocity by a polynomial of known degree in time (in practice, the degree has been taken equal to 0 or 1 ). The goodness of this approximation increases with the time sampling frequency which is limited by the rate at which we can perform stereo. Currently our hardware for stereo works at frequencies varying between 1 and $5 \mathrm{~Hz}$ depending upon the complexity of the scene. We have noticed that the use of noise in the state equation was very useful in that it helped compensating for the systematic modeling errors.

The third assumption is that the Kalman filter and more precisely the extended Kalman filter can be used to predict the positions and orientations of the straight line segments being tracked. Even though the assumptions that underlie the use of the Kalman filter are stretched a little in our case (but we have justified the reasons for our choice in Sections 4 and 6) we have found in our experiments that we obtained excellent results if the first assumption was well verified.

Several ideas developed in this paper are not completely new, and can be found in the literature (Roach and Aggarwal, 1979; Hwang, 1989; Crowley et al., 1988). Consider, for example, the concept of support. In (Hwang, 1989), Hwang postulates that the correspondence process in human vision is local and opportunistic. The correspondence of two image features in two consecutive frames should be determined only by the contextual information collected during some short time interval in the past. And the correspondence algorithm should allow multiple competing solutions. As more three-dimensional frames are observed, the correspondence that best fits the observed data should eventually win. Due to occlusion or absence of features or false match in the past, a trajectory may not find any match in the current frame by extension. In his algorithm, he uses a concept called age to indicate the number of times that a trajectory (similar to our concept of token) does not find any match in the observed frame. Any trajectory whose age is greater than 
MAXAGE (a fixed integer) is removed from further consideration.

Our concept of support differs significantly from the age concept in that it takes into account not only the number of times a token has not been present, but also the number of times it has been present in the past and how well the measurements agreed with the predictions. This is nicely summarized in the log-likelyhood function defined in Section 9.

In (Jenkin and Tsotsos, 1986), Jenkin and Tsotsos proposed an approach different from ours to handle the multiple matching problem. They called it a "wait and see" approach: multiple matches are first hypothesized to be correct and are later disambiguated based on a temporal smoothness constraint by considering all possible temporal combinations. Their approach requires to store data observed during the last instants (at least three frames) in memory. In our approach, only the $\log$-likelyhood function is updated and retained.

Our approach to tracking is similar to several methods which exist in the literature (Gennery, 1982; Broida and Chellappa, 1986; Broida and Chellappa, 1989) in the sense that they are also based on estimation theory and that the state parameters are estimated by filtering over time. The main difference is that the other approaches assume that there exists only one moving object in the scene or that all objects are known a priori. Such an assumption is not used in our approach in which ob jects are segmented after tracking, making it more flexible.

We are currently programming this technique on a special purpose hardware to accommodate the throughput of the stereo process and trying to extend it to deal with significantly curved objects.

\section{Appendix: Proofs of the Theorems}

In this appendix, we prove that when the angular velocity $\omega(t)$ is constant and the linear velocity $\mathbf{v}(t)$ is a polynomial in $t$ we can integrate in closed-form the differential equation 13 , i.e we can express the vector $\mathbf{p}_{t}$ as a function of $\omega(t), \mathbf{v}(t)$.

The following notation (exponential of a matrix) will be used in this appendix

$$
e^{M} \triangleq \mathrm{I}+\frac{1}{1 !} M+\frac{1}{2 !} M^{2}+\cdots+\frac{1}{n !} M^{n}+\cdots
$$

where $M$ is an $m \times m$ matrix, $\mathbf{I}$ is the $m \times m$ identity matrix, and $M^{n}$ denotes the multiplication of $n$ matrices $M$, i.e.,

$$
M^{n} \triangleq \overbrace{M \cdots M}^{n}
$$

If $M$ is a constant matrix, it can be easily shown that

$$
\frac{\mathrm{d}}{\mathrm{dt}} e^{M t}=M e^{M t}=e^{M t} M,
$$

since we have

$$
\frac{\mathrm{d}}{\mathrm{dt}}(M t)^{n}=\frac{\mathrm{d}}{\mathrm{dt}}\left(M^{n} t^{n}\right)=n M^{n} t^{n-1}=n M(M t)^{n-1}=n(M t)^{n-1} M .
$$

Note that in general, if $M$ is not a constant matrix, Equation 50 does not hold.

The following theorem known as Rodrigues' formula (Rodrigues, 1840) will be used in the following derivation. 
Theorem 3: Rodrigues' formula

Given a three-dimensional vector $\mathbf{r}$. The following relation holds:

$$
e^{\tilde{\mathbf{r}}}=\mathbf{I}_{3}+\frac{\sin \theta}{\theta} \tilde{\mathbf{r}}+\frac{1-\cos \theta}{\theta^{2}} \tilde{\mathbf{r}}^{2},
$$

where $\widetilde{\mathbf{r}}$ has the same definition as in Equation 12 and $\theta$ is the magnitude of $\mathbf{r}$ (i.e., $\theta=\|\mathbf{r}\|$ ).

Proof: From Equation 49, $e^{\widetilde{\mathbf{r}}}$ can be written as:

$$
e^{\tilde{\mathbf{r}}}=\mathrm{I}_{3}+\frac{1}{1 !} \tilde{\mathbf{r}}+\frac{1}{2 !} \tilde{\mathbf{r}}^{2}+\cdots+\frac{1}{n !} \tilde{\mathbf{r}}^{n}+\cdots \cdot
$$

It can be easily verified that

$$
\begin{aligned}
\widetilde{\mathbf{r}}^{2 n-1} & =(-1)^{n-1} \theta^{2(n-1)} \widetilde{\mathbf{r}}, \quad \text { for } n \geq 1 \\
\widetilde{\mathbf{r}}^{2 n} & =(-1)^{n-1} \theta^{2(n-1)} \widetilde{\mathbf{r}}^{2}, \quad \text { for } n \geq 1
\end{aligned}
$$

Therefore we have

$$
\begin{aligned}
e^{\tilde{\mathbf{r}}}=\mathrm{I}_{3} & +\left(\frac{1}{1 !}-\frac{\theta^{2}}{3 !}+\cdots+\frac{(-1)^{n-1} \theta^{2(n-1)}}{(2 n-1) !}+\cdots\right) \tilde{\mathbf{r}} \\
& +\left(\frac{1}{2 !}-\frac{\theta^{2}}{4 !}+\cdots+\frac{(-1)^{n-1} \theta^{2(n-1)}}{(2 n) !}+\cdots\right) \tilde{\mathbf{r}}^{2}
\end{aligned}
$$

Recall that

$$
\sin \theta=\frac{\theta}{1 !}-\frac{\theta^{3}}{3 !}+\frac{\theta^{5}}{5 !}+\cdots+(-1)^{n-1} \frac{\theta^{2 n-1}}{(2 n-1) !}+\cdots,
$$

and

$$
\cos \theta=1-\frac{\theta^{2}}{2 !}+\frac{\theta^{4}}{4 !}-\cdots+(-1)^{n} \frac{\theta^{2 n}}{(2 n) !}+\cdots,
$$

we thus get Formula 51.

Now we show the following important theorem.

Theorem 4: Trajectory with constant angular velocity and polynomial linear velocity

The trajectory of a point $\mathbf{p}_{t}$ given by Equation 13 can be given in closed form if the angular velocity is constant and if the translational velocity is a polynomial of degree $n(n \geq 0)$.

Proof: Let the motion be described by $\boldsymbol{\omega}_{t}=\boldsymbol{\omega}$ and

$$
\mathrm{v}_{t}=\mathrm{v}_{0}+\mathrm{v}_{1}\left(t-t_{0}\right)+\mathrm{v}_{2} \frac{\left(t-t_{0}\right)^{2}}{2 !}+\cdots+\mathrm{v}_{n} \frac{\left(t-t_{0}\right)^{n}}{n !}
$$

where $v_{0}$ is the velocity at time $t_{0}$. Define a new variable $y_{t}$ so that

$$
\mathbf{y}_{t}=e^{-\tilde{\omega}\left(t-t_{0}\right)} \mathbf{p}_{t}
$$


This yields $\mathbf{y}_{t_{0}}=\mathbf{p}_{0}$, the position of the point at $t_{0}$. Based on Equation 50, we have

$$
\dot{\mathbf{y}}_{t}=e^{-\widetilde{\boldsymbol{\omega}}\left(t-t_{0}\right)}\left(\dot{\mathbf{p}}_{t}-\tilde{\omega} \mathbf{p}_{t}\right)=e^{-\tilde{\omega}\left(t-t_{0}\right)} \mathbf{v}_{t}
$$

Integrating the above equation, we get

$$
\mathbf{y}_{t}=\mathbf{p}_{0}+\int_{t_{0}}^{t} e^{-\widetilde{\omega}\left(s-t_{0}\right)} \mathbf{v}_{s} d s
$$

From the definition of $y_{t}$, we thus have

$$
\mathbf{p}_{t}=e^{\widetilde{\boldsymbol{\omega}}\left(t-t_{0}\right)} \mathbf{y}_{t}=e^{\widetilde{\omega}\left(t-t_{0}\right)} \mathbf{p}_{0}+\int_{t_{0}}^{t} e^{\widetilde{\boldsymbol{\omega}}(t-s)} \mathbf{v}_{s} d s
$$

Using Theorem 3 , we find that

$$
e^{\tilde{\boldsymbol{\omega}}\left(t-t_{0}\right)}=W
$$

where $W$ is given by Equation 15. Denoting the last term of Equation 52 by $\mathbf{r}_{t}$ and using Theorem 3, we have

$$
\begin{aligned}
\mathbf{r}_{t}= & \int_{t_{0}}^{t}\left[\mathbf{I}_{3}+\frac{\sin [\theta(t-s)]}{\theta} \tilde{\boldsymbol{\omega}}+\frac{1-\cos [\theta(t-s)]}{\theta^{2}} \tilde{\omega}^{2}\right] \\
& {\left[\mathbf{v}_{0}+\mathbf{v}_{1}\left(s-t_{0}\right)+\mathbf{v}_{2} \frac{\left(s-t_{0}\right)^{2}}{2 !}+\cdots+\mathbf{v}_{n} \frac{\left(s-t_{0}\right)^{n}}{n !}\right] d s }
\end{aligned}
$$

Let us define

$$
L_{k}=\int_{t_{0}}^{t}\left(s-t_{0}\right)^{k} \sin [\theta(t-s)] d s
$$

and

$$
J_{k}=\int_{t_{0}}^{t}\left(s-t_{0}\right)^{k} \cos [\theta(t-s)] d s
$$

We can express $\mathbf{r}_{t}$ in terms of $L_{k}$ and $J_{k}(k=0 . . n)$. If we obtain closed-form expressions for $L_{k}$ and $J_{k}$, this will be true also for $\mathbf{r}_{t}$. Indeed,

$$
\begin{aligned}
L_{k} & =\frac{1}{\theta} \int_{t_{0}}^{t}\left(s-t_{0}\right)^{k} d(\cos [\theta(t-s)]) \\
& =\left.\frac{1}{\theta}\left(s-t_{0}\right)^{k} \cos [\theta(t-s)]\right|_{s=t_{0}} ^{s=t}-\frac{k}{\theta} \int_{t_{0}}^{t}\left(s-t_{0}\right)^{k-1} \cos [\theta(t-s)] d s \\
& =\frac{1}{\theta}\left(t-t_{0}\right)^{k}-\frac{k}{\theta} J_{k-1} \\
J_{k} & =-\frac{1}{\theta} \int_{t_{0}}^{t}\left(s-t_{0}\right)^{k} d(\sin [\theta(t-s)]) \\
& =-\left.\frac{1}{\theta}\left(s-t_{0}\right)^{k} \sin [\theta(t-s)]\right|_{s=t_{0}} ^{s=t}+\frac{k}{\theta} \int_{t_{0}}^{t}\left(s-t_{0}\right)^{k-1} \sin [\theta(t-s)] d s \\
& =\frac{k}{\theta} L_{k-1} \\
L_{0} & =\int_{t_{0}}^{t} \sin [\theta(t-s)] d s=\frac{1}{\theta}\left\{1-\cos \left[\theta\left(t-t_{0}\right)\right]\right\} \\
J_{0} & =\int_{t_{0}}^{t} \cos [\theta(t-s)] d s=\frac{1}{\theta} \sin \left[\theta\left(t-t_{0}\right)\right]
\end{aligned}
$$


By iteration, we can obtain closed forms of $L_{k}$ and $J_{k}$ for all $k>0$. Indeed, if we use the following notations:

$$
\begin{aligned}
\mathbf{x}_{k}=\left[\begin{array}{l}
L_{k} \\
J_{k}
\end{array}\right], \quad A & =\left[\begin{array}{cc}
0 & -1 \\
1 & 0
\end{array}\right], \quad \mathbf{b}_{k}=\left[\begin{array}{c}
\left(t-t_{0}\right)^{k} / \theta \\
0
\end{array}\right], \\
\text { and } \quad \mathbf{a} & =\left[\begin{array}{c}
1-\cos \left[\theta\left(t-t_{0}\right)\right] \\
\sin \left[\theta\left(t-t_{0}\right)\right]
\end{array}\right],
\end{aligned}
$$

we have

$$
\begin{aligned}
& \mathbf{x}_{k}=\frac{k}{\theta} A \mathbf{x}_{k-1}+\mathbf{b}_{k} \text { for } k>0 \\
& \mathbf{x}_{0}=\frac{1}{\theta} \mathbf{a} .
\end{aligned}
$$

After some algebra, we obtain

$$
\mathbf{x}_{k}=\frac{k !}{\theta^{k+1}} A_{k} \mathbf{a}+B_{k} \quad \text { for } k>0
$$

where

$$
\begin{aligned}
A_{k}= & (-1)^{\lceil(k+1) / 2]}\left[\begin{array}{cc}
(k+1) \bmod 2 & k \bmod 2 \\
-k \bmod 2 & (k+1) \bmod 2
\end{array}\right], \\
B_{k}= & {\left[\begin{array}{c}
\sum_{i=0}^{\lceil k / 2\rceil} \frac{(-1)^{i}}{\theta^{2 i+1}} \frac{\mathrm{d}^{2 i}}{\mathrm{~d} t^{2 i}}\left(t-t_{0}\right)^{k} \\
\sum_{i=0}^{[(k-1) / 2\rceil} \frac{(-1)^{i}}{\theta^{2(i+1)}} \frac{\mathrm{d}^{2 i+1}}{\mathrm{~d} t^{2 i+1}}\left(t-t_{0}\right)^{k}
\end{array}\right] . }
\end{aligned}
$$

Here $\lceil j\rceil$ denotes the largest integer less than $j$, "mod" denotes the modulo function, and $\frac{\mathrm{d}^{i}}{\mathrm{~d} \tau^{i}}(\cdot)$ denotes the $i$-th derivative with respect to $t$. This yields a closed-form expression for $\mathbf{r}_{t}$ and therefore, also for $\mathbf{p}_{t}$.

Q.E.D.

Theorem 1 is a special case of Theorem 4 for $\mathrm{v}_{t}=\mathrm{v}$ (i.e., $n=0$ ). The reader can easily verify it.

Theorem 2 describes the special case $\mathbf{v}_{t}=\mathbf{v}+\mathbf{a}\left(t-t_{0}\right)$ (i.e., $\left.n=1\right)$. From Equation 59, we obtain:

$$
\begin{aligned}
L_{1} & =\frac{1}{\theta}\left(t-t_{0}\right)-\frac{1}{\theta^{2}} \sin \left[\theta\left(t-t_{0}\right)\right]=\frac{1}{\theta^{2}}\left\{\theta\left(t-t_{0}\right)-\sin \left[\theta\left(t-t_{0}\right)\right]\right\} \\
J_{1} & =\frac{1}{\theta^{2}}\left\{1-\cos \left[\theta\left(t-t_{0}\right)\right]\right\}
\end{aligned}
$$

After some algebra, we get Theorem 2. 


\section{References}

J.K. Aggarwal and Y.F. Wang. Analysis of a sequence of images using point and line correspondences. In Proc. International Conference on Robotics and Automation, pages 1275-1280, IEEE, Raleigh, NC, March 31-April 31987.

N. Ayache. Construction et Fusion de Représentations Visuelles 3D - Applications à la Robotique Mobile. PhD thesis, University of Paris XI, Paris-Orsay, 1988. Thèse d'Etat.

N. Ayache and O.D. Faugeras. Building, registrating and fusing noisy visual maps. In Proc. First International Conference on Computer Vision, pages 73-82, IEEE, London, UK, June 1987.

N. Ayache and O.D. Faugeras. Maintaining representations of the environment of a mobile robot. In International Symposium on Robotics Research, MIT Press, August 1987. Santa-Cruz, California.

Nicholas Ayache and Olivier D. Faugeras. Maintaining Representations of the Environment of a Mobile Robot. IEEE transactions on Robotics and Automation, 5(6):804-819, December 1989. also INRIA report 789 .

N. Ayache and F. Lustman. Fast and reliable passive trinocular stereovision. In Proc. First International Conference on Computer Vision, pages 422-427, IEEE, London, UK, June 1987.

H. Baker and T.O. Binford. Depth from edge and intensity based stereo. In Proceedings 7th Joint Conference on Artificial Intelligence, Vancouver, Canada, pages 631-636, August 1981.

Y. Bar-Shalom and T.E. Fortmann. Tracking and Data Association. Academic Press, 1988.

T.J. Broida and R. Chellappa. Kinematics and structure of a rigid object from a sequence of noisy images. In Proc. IEEE Workshop on Motion: Representation and Analysis, pages 95-100, IEEE, Charleston, SC, May 1986.

T.J. Broida and R. Chellappa. Experiments and uniqueness results on object structure and kinematics from a sequences of monocular images. In Proc. IEEE Workshop on Visual Motion, pages 21-30, IEEE, Irvine, CA, March 1989.

J.L. Crowley, P. Stelmaszyk, and C. Discours. Measuring image flow by tracking edge-lines. In Proc. Second International Conference on Computer Vision, pages 658-664, IEEE, Tampa, Florida, December 1988.

R. Deriche and O. Faugeras. Tracking line segments. In O. Faugeras, editor, Proc. European Conference on Computer Vision, pages 259-268, Springer-Verlag, Antibes, France, April 1990.

E.D. Dickmanns. 4D-dynamic scene analysis with integral spatio-temporal models. In Proc. ISSR'87, pages 73-80, Santa-Cruz, 1987.

E.D. Dickmanns and V. Graefe. Applications of dynamic monocular machine vision. Machine Vision and Applications, 1:241-261, 1988.

E.D. Dickmanns and V. Graefe. Dynamic monocular machine vision. Machine Vision and Applications, 1:223-240, 1988.

H.F. Durrant-Whyte. Integration, Coordination, and Control of Multi-sensor. Kluwer Academic Publishers, 1988. 
O.D. Faugeras. Three-Dimensional Computer Vision. MIT Press, 1991. To appear.

O.D. Faugeras and G. Toscani. The calibration problem for stereo. In Proc. IEEE Conference on Computer Vision and Pattern Recognition, pages 15-20, IEEE, Miami Beach, Florida, June 1986.

O.D. Faugeras, N. Ayache, and Z. Zhang. A preliminary in vestigation of the problem of determining ego- and object motions from stereo. In Proc. 9th International Conference on Pattern Recognition, pages $242-246$, Rome, Italy, 1988.

O.D. Faugeras, R. Deriche, N. Ayache, F. Lustman, and E. Giuliano. Depth and Motion Analysis: the machine being developed within Esprit Project 940. In Proceedings of the IAPR Workshop on Computer Vision (Special Hardware and Industrial Applications), pages 35-44, October 1988. Tokyo, Japan.

Olivier D. Faugeras. On the motion of 3-D curves and its relationship to optical flow. In Proceedings of the 1st ECCV, pages 107-117, Springer Verlag, April 1990.

Olivier D. Faugeras and Steven Maybank. Motion from point matches: multiplicity of solutions. The International Journal of Computer Vision, 4(3):225-246, June 1990. also INRIA Tech. Report 1157.

Olivier D. Faugeras, Elizabeth Lebras-Mehlman, and Jean-Daniel Boissonnat. Representing Stereo data with the Delaunay Triangulation. Artificial Intelligence Journal, 44(1-2), July 1990. also INRIA Tech. Report 788.

J.P. Gambotto. Tracking points and line segments in image sequences. In Proc. IEEE Workshop on Visual Motion, pages 38-45, IEEE, Irvine, California, March 20-22 1989.

D.B. Gennery. Tracking known three-dimensional objects. In Proc. of AAAI-82, pages 13-17, Carnegie-Mellon University, Pittsburgh, 1982.

J.J. Gibson. The Perception of the Visual World. Houghton Mifflin, Boston, Mass., 1950.

G.L. Gordon. On the tracking of featureless objects with occlusion. In Proc. IEEE Workshop on Visual Motion, pages 13-20, IEEE, Irvine, California, March 20-22 1989.

W.E.L. Grimson. From Images to Surfaces. MIT Press : Cambridge, 1981.

W.E.L. Grimson. Computational experiments with a feature based stereo algorithm. IEEE Transactions on Pattern Analysis and Machine Intelligence, 7, No 1:17-34, 1985.

Ellen C. Hildreth. The Measurement of Visual Motion. MIT Press, Cambridge, Mass., 1984.

Berthold K. P. Horn and Brian G. Schunk. Determining Optical Flow. Artificial Intelligence, 17:185-203, 1981.

T.S. Huang and R.Y. Tsai. Image sequence analysis: motion estimation. In T.S. Huang, editor, Image Sequence Processing and Dynamic Scene Analysis, NY: Springer-Verlag, New York, 1981.

V.S.S. Hwang. Tracking feature points in time-varying images using an opportunistic selection approach. Pattern Recognition, 22(3):247-256, 1989.

M. Jenkin and J.K. Tsotsos. Applying temporal constraints to the dynamic stereo problem. Computer Vision, Graphics, and Image Processing, 24:16-32, 1986. 
Y.C. Kim and J.K. Aggarwal. Determining object motion in a sequence of stereo images. IEEE Journal of Robotics and Automation, 3(6):599-614, December 1987.

Yoshifumi Kitamura and Masahiko Yachida. Three-dimensional data acquisition by trinocular vision. Advanced Robotics, 4(1):29-42, 1990. Robotics Society of Japan.

Jan J. Koenderink. Optic Flow. Vision Research, 26(1):161-180, 1986.

Jan J. Koenderink and A. J. van Doorn. Invariant properties of the motion parallax field due to the movement of rigid bodies relative to an observer. Optica Acta, 22:717-723, 1975.

Jan J. Koenderink and A. J. van Doorn. How an ambulant observer can construct a model of the environment from the geometrical structure of the visual inflow. Oldenburg, Muenchen, 1978.

Y. Liu and T.S. Huang. Estimation of rigid body motion using straight line correspondences. In Proceedings Workshop on Motion: Representation and Analysis, Charleston, South Carolina, USA, pages 47-51, IEEE, May 1986.

Y. Liu and T.S. Huang. A linear algorithm for determining motion and structure from line correspondences. Computer Vision, Graphics, and Image Processing, 44(1):35-57, 1988.

H.C. Longuet-Higgins. A computer algorithm for reconstructing a scene from two projections. Nature, 293:133-135, 1981.

B. Lowerre and R. Reddy. The harpy speech understanding system. In W. Lea, editor, Trends in Speech Recognition, N.J.: Prentice-Hall, Englewood Cliffs, 1980.

D. Marr and T. Poggio. Cooperative computation of stereo disparity. Science, 194:283-287, 1976.

D. Marr and T. Poggio. A computational theory of human stereo vision. Proc. R. Soc., B-204:301$328,1979$.

P.S. Maybeck. Stochastic Models, Estimation and Control. Volume 1, Academic Press, 1979.

P.S. Maybeck. Stochastic Models, Estimation and Control. Volume 2, Academic Press, 1982.

H.H Nagel. Displacement Vectors Derived from Second Order Intensity Variations in Image Sequences. Computer Vision, Graphics, and Image Processing, 21:85-117, 1983.

H.H. Nagel. Image Sequences - Ten (octal) Years - From Phenomenology towards a Theoretical Foundation. In Proceedings 8th ICPR, pages 1174-1185, IEEE, Paris, France, October 1986.

H.K. Nishihara. PRISM, a Practical Real-Time Imaging Stereo Matcher. Technical Report A.I. Memo 780, MIT, Cambridge, MA, 1984. 31 pages.

Y. Ohta and T. Kanade. Stereo by intra- and inter-scanline search. IEEE Transactions on PAMI, 7, No 2:139-154, 1985.

S.B. Pollard, J.E.W. Mayhew, and J.P. Frisby. PMF : a stereo correspondence algorithm using a disparity gradient constraint. Perception, 14:449-470, 1985.

F. Preparata and M. Shamos. Computational Geometry, An Introduction. Springer-Verlag, NewYork, 1986. 
J.W. Roach and J.K. Aggarwal. Computer tracking of objects moving in space. IEEE Trans. on Pattern Analysis and Machine Intelligence, 1(2):127-135, 1979.

K.S. Roberts. A new representation for a line. In Proc. of the 1988 Conference on Computer Vision and Pattern Recognition, pages 635-640, IEEE, Ann Arbor, Michigan', June 5-9 1988.

O. Rodrigues. Des lois géométriques qui régissent les déplacements d'un système solide dans l'espace, et de la variation des coordonnées provenant de ces déplacements considérés indépendamment des causes qui peuvent les produire. Journal de Mathématiques Pures et Appliquées, $5: 380-440,1840$.

S.K. Sethi and R. Jain. Finding trajectories of feature points in a monocular image sequence. IEEE Trans. on Pattern Analysis and Machine Intelligence, 9(1):56-73, 1987.

R.Y. Tsai and T.S. Huang. Estimating 3-D motion parameters of a rigid planar patch, i. IEEE Trans. on Acoustic, Speech and Signal Processing, 29(6):1147-1152, December 1981.

R.Y. Tsai and T.S. Huang. Uniqueness and estimation of three-dimensional motion parameters of rigid objects with curved surface. IEEE Trans. on Pattern Analysis and Machine Intelligence, 6(1):13-26, Jan. 1984.

Shimon Ullman. The Interpretation of Visual Motion. MIT Press, 1979.

J.A. Webb and J.K. Aggarwal. Structure from motion of rigid and jointed objects. Artificial Intelligence, 19:107-130, 1982.

J. Weng, T.S. Huang, and N. Ahuja. 3-D motion estimation, understanding, and prediction from noisy image sequences. IEEE Trans. on Pattern Analysis and Machine Intelligence, 9(3):370-389, 1987.

M. Yachida. 3D Data Acquisition by Multiple Views. In O.D. Faugeras and G. Giralt, editors, Robotics Research: the Third International Symposium, pages 11-18, MIT Press, Cambridge, Mass., 1986.

B.L. Yen and T.S. Huang. Determining 3-D motion/structure of a rigid body over 3 frames using straight line correspondences. In Proc. IEEE Conference on Computer Vision and Pattern Recognition, pages 267-272, IEEE, Washington, DC, June 19-23 1983.

G.S. Young and R. Chellappa. 3-D motion estimation using a sequence of noisy stereo images. In Proc. IEEE Conf. Computer Vision and Pattern Recognition, pages 710-716, IEEE, 1988.

Zhengyou Zhang. Motion Analysis from a Sequence of Stereo Frames and its Applications. PhD thesis, University of Paris-Sud, Orsay, Paris, France, 1990. in English.

Z. Zhang and O.D. Faugeras. Building a 3D world model with a mobile robot: 3D line segment representation and integration. In Proc. 10th International Conference on Pattern Recognition, pages 38-42, IEEE, Atlantic City, New Jersey, USA, June 1990.

Z. Zhang and O.D. Faugeras. Tracking and motion estimation in a sequence of stereo frames. In L.C. Aiello, editor, Proc. the 9th European Conference on Artificial Intelligence, pages 747-752, Stockholm, Sweden, August 1990. 
Z. Zhang and O.D. Faugeras. Determining motion from 3D line segments: a comparative study. Image and Vision Computing, 9(1):10-19, February 1991.

Z. Zhang and O.D. Faugeras. Estimation of displacements from two 3D frames obtained from stereo. IEEE Trans. Pattern Analysis and Machine Intelligence, September 1991. accepted, to appear.

Z. Zhang, O.D. Faugeras, and N. Ayache. Analysis of a sequence of stereo scenes containing multiple moving objects using rigidity constraints. In Proc. Second International Conference on Computer Vision, pages 177-186, IEEE, Tampa, Florida, December 1988.

X. Zhuang and R.M. Haralick. Two view motion analysis. In Proc. IEEE Conf. Computer Vision and Pattern Recognition, pages 686-690, San Francisco, California, June 1985. 
ISSN $0249-6399$ 